\title{
Model-Checking Structured Context-Free Languages
}

\author{
Michele Chiari $^{1(\bowtie)}\left(\mathbb{D}\right.$, Dino Mandrioli ${ }^{1}$ (D), and Matteo Pradella ${ }^{1,2}$ (D) \\ 1 DEIB, Politecnico di Milano, Milan, Italy \\ \{michele.chiari, dino.mandrioli, \\ matteo.pradella\}@polimi.it \\ 2 IEIIT, Consiglio Nazionale delle Ricerche, Milan, Italy

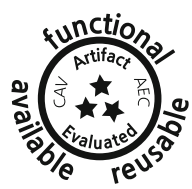

\begin{abstract}
The problem of model checking procedural programs has fostered much research towards the definition of temporal logics for reasoning on context-free structures. The most notable of such results are temporal logics on Nested Words, such as CaRet and NWTL. Recently, the logic OPTL was introduced, based on the class of Operator Precedence Languages (OPL), more powerful than Nested Words. We define the new OPL-based logic POTL, and provide a model checking procedure for it. POTL improves on NWTL by enabling the formulation of requirements involving pre/post-conditions, stack inspection, and others in the presence of exception-like constructs. It improves on OPTL by being FO-complete, and by expressing more easily stack inspection and function-local properties. We developed a model checking tool for POTL, which we experimentally evaluate on some interesting use-cases.
\end{abstract}

Keywords: Linear temporal logic • Operator precedence languages • Model Checking $\cdot$ Visibly pushdown languages $\cdot$ Input-driven languages

\section{Introduction}

Model checking is one of the most successful techniques for the verification of software programs. It consists in the exhaustive verification of the mathematical model of a program against a specification of its desired behavior. The kind of properties that can be proved in this way depends both on the formalism employed to model the program, and on the one used to express the specification. The initial and most classical frameworks consist in the use of operational formalisms, such as Transition Systems and Finite State Automata (generally Büchi automata) for the model, and temporal logics such as Linear-time Temporal Logic (LTL), Computation-Tree Logic (CTL) and CTL* for the specification [24]. The success of such logics is due to their ease in reasoning about linear or branching sequences of events over time, by expressing liveness and safety properties, their conciseness with respect to automata, and the complexity of their model checking.

In this paper we consider linear-time temporal domains. LTL limits its set of expressible properties to the First-Order Logic (FOL) definable fragment

(C) The Author(s) 2021

A. Silva and K. R. M. Leino (Eds.): CAV 2021, LNCS 12760, pp. 387-410, 2021.

https://doi.org/10.1007/978-3-030-81688-9_18 
of regular languages. This is quite restrictive when compared with the most popular abstract models of procedural programs, such as Pushdown Systems, Boolean Programs [10], and Recursive State Machines [3]. All such stack-based formalisms show behaviors that are expressible by means of Context-Free Languages (CFL), rather than regular ones. State and configuration reachability, fair computation problems, and model checking of regular specifications have been thoroughly studied for such formalisms $[3,4,13,17,28,30,32,40,51,55]$. To expand the expressive power of specification languages too, $[12,14]$ augmented LTL with Presburger arithmetic constraints on the occurrences of states, obtaining a logic capable of even some context-sensitive specifications, but with only restricted decidable fragments. [41] introduced model checking of pushdown tree automata specifications on regular systems, and Dynamic Logic was extended to some limited classes of CFL [34]. Decision procedures for different kinds of regular constraints on stack contents have been given in $[18,29,37]$.

A coherent approach came with the introduction of temporal logics based on Visibly Pushdown Languages (VPL) [7], a.k.a. Input-Driven Languages [47]. Such logics, namely CaRet [6] and its FO-complete successor NWTL [2], model the execution trace of a procedural program as a Nested Word [8], consisting in a linear ordering augmented with a one-to-one matching relation between function calls and returns. They are the first ones featuring temporal modalities that explicitly refer to the nesting structure of CFL [4]. This enables requirement specifications to include Hoare-style pre/post-conditions, stack-inspection properties, and more. A $\mu$-calculus based on VPL extends model checking to branching-time semantics in [5], while [16] introduces a temporal logic capturing the whole class of VPL. Timed extensions of CaRet are given in [15].

VPL too have their limitations. They are more general than Parenthesis Languages [46], but their matching relation is essentially constrained to be one-toone [43]. This hinders their suitability to model processes in which a single event must be put in relation with multiple ones. Unfortunately, computer programs often present such behaviors: exceptions and continuations are single events that cause the termination (or re-instantiation) of multiple functions on the stack.

To reason about such behaviors, temporal logics based on Operator Precedence Languages (OPL) have been proposed [22]. OPL were initially introduced with the purpose of efficient parsing [31], a field in which they continue to offer useful applications [11]. They are capable of capturing the syntax of arithmetic expressions, and other constructs whose context-free structure is not immediately visible. The generality of the structure of their syntax trees is much greater than that of VPL, which are strictly included in OPL [25]. Nevertheless, they retain the same closure properties that make regular languages and VPL suitable for automata-theoretic model checking: OPL are closed under Boolean operations, concatenation, Kleene *, and language emptiness and inclusion are decidable [42]. They have been characterized by means of push-down automata, Monadic Second-Order Logic and, recently, by an extension of Regular Expressions [42,44].

OPTL [22] is the first linear-time temporal logic for which a model checking procedure has been given on both finite and $\omega$-words of OPL. It enables reasoning 
on procedural programs with exceptions, expressing properties about whether a function can be terminated by an exception, or throw one, and also pre/postconditions. NWTL can be translated into OPTL in linear time, thus the latter is capable of expressing all properties that can be formalized in CaRet and NWTL, and many more. [22] does not explore OPTL's expressiveness further, and does not investigate the practical applicability of their model checking construction.

In this article, we introduce Precedence Oriented Temporal Logic (POTL), which redefines the syntax and semantics of OPTL to be much closer to the context-free structure of words. With POTL, it is much easier to navigate a word's syntax tree, expressing requirements that are aware of its structure. From a more theoretical point of view, POTL is FO-complete whereas OPTL is not, so that CaRet, NWTL, OPTL and POTL constitute a strict hierarchy in terms of expressive power. Such a theoretical elaboration, however, is technically involved; thus, for length reasons, it is documented in a technical report [23].

In this paper, instead, we focus on the model-checking application of POTL. We provide a tableaux-construction procedure for model checking POTL, which yields nondeterministic automata of size at most singly exponential in the formula's length, and is thus not asymptotically greater than that of LTL, NWTL and OPTL. We implemented such a procedure in a tool called POMC, which we evaluate on several interesting case studies. POMC's performance is promising: almost all case studies are verified in seconds and with a reasonable memory consumption, with very few outliers. Such outliers are inevitable, due to the exponential complexity of the task.

The related work on tools is not as rich as the theoretical one. Tools and libraries such as VPAlib [48], VPAchecker [54], OpenNWA [27] and SymbolicAutomata [26] only implement operations such as union, intersection, universality/inclusion/emptiness check for Visibly Pushdown or Nested Word Automata, but have no model checking capabilities. PAL [19] uses nested-word based monitors to express program specifications, and a tool based on BLAST [36] implements its runtime monitoring and model checking. PAL follows the paradigm of program monitors, and is not - strictly speaking - a temporal logic. PTCaRet [52] is a past version of CaRet, and its runtime monitoring has been implemented in JavaMOP [20]. [49,50] describe a tool for model checking programs against CaRet specifications. Since its purpose is malware detection, it targets program binaries directly by modeling them as Pushdown Systems. Unfortunately, this tool does not seem to be available online. To the best of our knowledge, POMC is the only publicly-available ${ }^{1}$ tool for model-checking temporal logics capable of expressing context-free properties.

The paper is organized as follows: we give some background on OPL in Sect. 2, we introduce POTL in Sect. 3 and its model checking in Sect. 4, and we evaluate our prototype model checker in Sect. 5. Due to space constraints, we leave all formal proofs to a technical report [21].

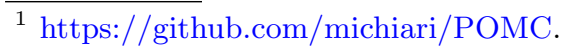




\section{Operator Precedence Languages}

We assume some familiarity with classical formal language theory concepts such as context-free grammar, parsing, shift-reduce algorithm, syntax tree (ST) $[33,35]$. Operator Precedence Languages (OPL) are usually defined through their generating grammars [31]; in this paper, however, we characterize them through their accepting automata [42] which are the natural way for stating equivalence properties with logic characterization, and for model checking. Readers not familiar with OPL may refer to [43] for more explanations on the following basic concepts; an explanatory example is also given at the end of this section.

Let $\Sigma$ be a finite alphabet, and $\varepsilon$ the empty string. We use a special symbol $\# \notin \Sigma$ to mark the beginning and the end of any string. An operator precedence matrix (OPM) $M$ over $\Sigma$ is a partial function $(\Sigma \cup\{\#\})^{2} \rightarrow\{\lessdot, \doteq,>\}$, that, for each ordered pair $(a, b)$, defines the precedence relation $(\mathrm{PR}) M(a, b)$ holding between $a$ and $b$. If the function is total we say that $\mathrm{M}$ is complete. We call the pair $(\Sigma, M)$ an operator precedence alphabet. Relations $\lessdot, \doteq,>$, are respectively named yields precedence, equal in precedence, and takes precedence. By convention, the initial \# yields precedence, and other symbols take precedence on the ending \#. If $M(a, b)=\pi$, where $\pi \in\{\lessdot, \doteq,>\}$, we write $a \pi b$. For $u, v \in \Sigma^{+}$we write $u \pi v$ if $u=x a$ and $v=b y$ with $a \pi b$. The role of $\mathrm{PR}$ is to give structure to words: they can be seen as special and more concise parentheses, where e.g. one "closing" $\rightarrow$ can match more than one "opening" $\lessdot$. Despite their graphical appearance, $\mathrm{PR}$ are not ordering relations.

Definition 1. An operator precedence automaton (OPA) is a tuple $\mathcal{A}=$ $(\Sigma, M, Q, I, F, \delta)$ where: $(\Sigma, M)$ is an operator precedence alphabet, $Q$ is a finite set of states (disjoint from $\Sigma$ ), $I \subseteq Q$ is the set of initial states, $F \subseteq Q$ is the set of final states, $\delta \subseteq Q \times(\Sigma \cup Q) \times Q$ is the transition relation, which is the union of the three disjoint relations $\delta_{\text {shift }} \subseteq Q \times \Sigma \times Q, \delta_{\text {push }} \subseteq Q \times \Sigma \times Q$, and $\delta_{\text {pop }} \subseteq Q \times Q \times Q$. An $O P A$ is deterministic iff $I$ is a singleton, and all three components of $\delta$ are-possibly partial-functions.

To define the semantics of OPA, we need some new notations. Letters $p, q, p_{i}, q_{i}, \ldots$ denote states in $Q$. We use $q_{0} \stackrel{a}{\longrightarrow} q_{1}$ for $\left(q_{0}, a, q_{1}\right) \in \delta_{\text {push }}, q_{0} \stackrel{a}{\rightarrow} \rightarrow q_{1}$ for $\left(q_{0}, a, q_{1}\right) \in \delta_{\text {shift }}, q_{0} \stackrel{q_{2}}{\longrightarrow} q_{1}$ for $\left(q_{0}, q_{2}, q_{1}\right) \in \delta_{\text {pop }}$, and $q_{0} \stackrel{w}{\sim} q_{1}$, if the automaton can read $w \in \Sigma^{*}$ going from $q_{0}$ to $q_{1}$. Let $\Gamma=\Sigma \times Q$ and $\Gamma^{\prime}=\Gamma \cup\{\perp\}$ be the stack alphabet; we denote symbols in $\Gamma^{\prime}$ as $[a, q]$ or $\perp$. We set $\operatorname{smb}([a, q])=a$, $\operatorname{smb}(\perp)=\#$, and $s t([a, q])=q$. For a stack content $\gamma=\gamma_{n} \ldots \gamma_{1} \perp$, with $\gamma_{i} \in \Gamma$, $n \geq 0$, we set $\operatorname{smb}(\gamma)=\operatorname{smb}\left(\gamma_{n}\right)$ if $n \geq 1, \operatorname{smb}(\gamma)=\#$ if $n=0$.

A configuration of an OPA is a triple $c=\langle w, q, \gamma\rangle$, where $w \in \Sigma^{*} \#, q \in Q$, and $\gamma \in \Gamma^{*} \perp$. A computation or run is a finite sequence $c_{0} \vdash c_{1} \vdash \ldots \vdash c_{n}$ of moves or transitions $c_{i} \vdash c_{i+1}$. There are three kinds of moves, depending on the PR between the symbol on top of the stack and the next input symbol:

Push move: if $\operatorname{smb}(\gamma) \lessdot a$ then $\langle a x, p, \gamma\rangle \vdash\langle x, q,[a, p] \gamma\rangle$, with $(p, a, q) \in$ $\delta_{\text {push }}$;

Shift move: if $a \doteq b$ then $\langle b x, q,[a, p] \gamma\rangle \vdash\langle x, r,[b, p] \gamma\rangle$, with $(q, b, r) \in \delta_{\text {shift }}$; Pop move: if $a>b$ then $\langle b x, q,[a, p] \gamma\rangle \vdash\langle b x, r, \gamma\rangle$, with $(q, p, r) \in \delta_{\text {pop }}$. 


\begin{tabular}{|c|c|c|c|c|c|}
\hline & \multicolumn{4}{|c|}{ call ret han exc } & \multirow{5}{*}{$\#[$ call $[[[$ han $[$ call $[$ call $[$ call $]]]$ exc $]$ call ret $]$ call ret $]$ ret $] \#$} \\
\hline call & $\lessdot$ & $\dot{\doteq}$ & $\lessdot$ & $>$ & \\
\hline ret & $\rightarrow$ & $>$ & $>$ & $>$ & \\
\hline han & $\lessdot$ & $>$ & $\lessdot$ & $\doteq$ & \\
\hline exc & $\rightarrow$ & $\rightarrow$ & $>$ & $>$ & \\
\hline
\end{tabular}

Fig. 1. OPM $M_{\text {call }}$ (left) and a string with chains shown by brackets (right).

Shift and pop moves are not performed when the stack contains only $\perp$. Push moves put a new element on top of the stack consisting of the input symbol together with the current state of the OPA. Shift moves update the top element of the stack by changing its input symbol only. Pop moves remove the element on top of the stack, and update the state of the OPA according to $\delta_{\text {pop }}$ on the basis of the current state of the OPA and the state of the removed stack symbol. They do not consume the input symbol, which is used only to establish the $>$ relation, remaining available for the next move. The OPA accepts the language $L(\mathcal{A})=\left\{x \in \Sigma^{*} \mid\left\langle x \#, q_{I}, \perp\right\rangle \vdash^{*}\left\langle \#, q_{F}, \perp\right\rangle, q_{I} \in I, q_{F} \in F\right\}$.

We now introduce the concept of chain, which makes the connection between OP relations and context-free structure explicit, through brackets.

Definition 2. A simple chain ${ }^{c_{0}}\left[c_{1} c_{2} \ldots c_{\ell}\right]^{c_{\ell+1}}$ is a string $c_{0} c_{1} c_{2} \ldots c_{\ell} c_{\ell+1}$, such that: $c_{0}, c_{\ell+1} \in \Sigma \cup\{\#\}, c_{i} \in \Sigma$ for every $i=1,2, \ldots \ell(\ell \geq 1)$, and $c_{0} \lessdot c_{1} \doteq$ $c_{2} \ldots c_{\ell-1} \doteq c_{\ell}>c_{\ell+1}$. A composed chain is a string $c_{0} s_{0} c_{1} s_{1} c_{2} \ldots c_{\ell} s_{\ell} c_{\ell+1}$, where $c_{0}\left[c_{1} c_{2} \ldots c_{\ell}\right]^{c_{\ell+1}}$ is a simple chain, and $s_{i} \in \Sigma^{*}$ is the empty string or is such that ${ }^{c_{i}}\left[s_{i}\right]^{c_{i+1}}$ is a chain (simple or composed), for every $i=0,1, \ldots, \ell(\ell \geq 1)$. Such a composed chain will be written as ${ }^{c_{0}}\left[s_{0} c_{1} s_{1} c_{2} \ldots c_{\ell} s_{\ell}\right]^{c_{\ell+1}} . c_{0}$ (resp. $\left.c_{\ell+1}\right)$ is called its left (resp. right) context; all symbols between them form its body.

A finite word $w$ over $\Sigma$ is compatible with an OPM $M$ iff for each pair of letters $c, d$, consecutive in $w, M(c, d)$ is defined and, for each substring $x$ of \#w\# that is a chain of the form ${ }^{a}[y]^{b}, M(a, b)$ is defined.

Chains can be identified through the traditional operator precedence parsing algorithm. We apply it to the sample word $w_{e x}=$ call han call call exc call ret ret, which is compatible with $M_{\text {call }}$ (for a more complete treatment, cf. [33,43]). First, write all precedence relations between consecutive characters, according to $M_{\text {call }}$. Then, recognize all innermost patterns of the form $a \lessdot c \doteq \ldots \doteq c>b$ as simple chains, and remove their bodies. Then, write the precedence relations between the left and right contexts of the removed body, $a$ and $b$, and iterate this process until only \#\# remains. This procedure is applied to $w_{e x}$ as follows:

$$
\begin{aligned}
& 1 \mid \# \lessdot \text { call } \lessdot \text { han } \lessdot \text { call } \lessdot \text { call }>\text { exc }>\text { call } \doteq \text { ret }>\text { ret }>\# \\
& 2 \# \lessdot \text { call } \lessdot \text { han } \lessdot \text { call }>\text { exc }>\text { call } \doteq \text { ret }>\text { ret }>\# \\
& 3 \text { \# } \lessdot \text { call } \lessdot \underline{\text { han }} \doteq \underline{\text { exc }}>\text { call } \doteq \text { ret }>\text { ret }>\# \\
& 4 \text { \# }<\text { call } \lessdot \underline{\text { call }} \doteq \underline{\text { ret }}>\text { ret }>\# \\
& 5 \# \lessdot \underline{\text { call }} \doteq \underline{\text { ret }}>\# \\
& 6 \# \doteq \#
\end{aligned}
$$


The chain body removed in each step is underlined. In step $1,{ }^{\text {call }}[\underline{\text { call }}]^{\text {exc }}$ is a simple chain, so its body call is removed. Then, in step 2 we recognize the simple chain han $[\underline{\text { call }}]^{\text {exc }}$, which means han $[\text { call }[\text { call }]]^{\text {exc }}$, where $[$ call $]$ is the chain body removed in step 1, is a composed chain. This way, we recognize, e.g., han $[\text { call }]^{\text {exc }},{ }^{\text {call }}[\text { han exc }]^{\text {call }}$ as simple chains, and han $[\text { call }[\text { call }]]^{\text {exc }}$ and call $[\text { han }[\text { call }[\text { call }]] \text { exc }]^{\text {call }}$ as composed chains (with inner chain bodies enclosed in brackets). Figure 1 shows the structure of a longer version of $w_{e x}$, which is an isomorphic representation of its ST as depicted in Fig. 4. Each chain corresponds to an internal node, and the fringe of the subtree rooted at it is the chain's body.

Let $\mathcal{A}$ be an OPA. We call a support for the simple chain ${ }^{c_{0}}\left[c_{1} c_{2} \ldots c_{\ell}\right]^{c_{\ell+1}}$ any path in $\mathcal{A}$ of the form $q_{0} \stackrel{c_{1}}{\longrightarrow} q_{1} \rightarrow \ldots \stackrel{-\rightarrow}{\rightarrow} q_{\ell-1} \stackrel{c_{\ell}}{\rightarrow} q_{\ell} \stackrel{q_{0}}{\Longrightarrow} q_{\ell+1}$. The label of the last (and only) pop is exactly $q_{0}$, i.e. the first state of the path; this pop is executed because of relation $c_{\ell}>c_{\ell+1}$. We call a support for the composed chain $^{c_{0}}\left[s_{0} c_{1} s_{1} c_{2} \ldots c_{\ell} s_{\ell}\right]^{c_{\ell+1}}$ any path in $\mathcal{A}$ of the form $q_{0} \stackrel{s_{0}}{\longrightarrow} q_{0}^{\prime} \stackrel{c_{1}}{\longrightarrow} q_{1} \stackrel{s_{1}}{\longrightarrow} q_{1}^{\prime} \stackrel{c_{2}}{\rightarrow}$ $\ldots \stackrel{c_{\ell}}{\rightarrow} q_{\ell} \stackrel{s_{\ell}}{\longrightarrow} q_{\ell}^{\prime} \stackrel{q_{0}^{\prime}}{\longrightarrow} q_{\ell+1}$ where, for every $i=0,1, \ldots, \ell$ : if $s_{i} \neq \epsilon$, then $q_{i} \stackrel{s_{i}}{\longrightarrow} q_{i}^{\prime}$ is a support for the chain ${ }^{c_{i}}\left[s_{i}\right]^{c_{i+1}}$, else $q_{i}^{\prime}=q_{i}$.

Chains fully determine the parsing structure of any OPA over $(\Sigma, M)$. If the OPA performs the computation $\left\langle s b, q_{i},\left[a, q_{j}\right] \gamma\right\rangle \vdash^{*}\left\langle b, q_{k}, \gamma\right\rangle$, then ${ }^{a}[s]^{b}$ is necessarily a chain over $(\Sigma, M)$, and there exists a support like the one above with $s=s_{0} c_{1} \ldots c_{\ell} s_{\ell}$ and $q_{\ell+1}=q_{k}$. This corresponds to the parsing of the string $s_{0} c_{1} \ldots c_{\ell} s_{\ell}$ within the contexts $a, b$, which contains all information needed to build the subtree whose frontier is that string.

Consider the OPA $\mathcal{A}(\Sigma, M)=\left(\Sigma, M,\{q\},\{q\},\{q\}, \delta_{\max }\right)$ where $\delta_{\max }(q, q)=$ $q$, and $\delta_{\max }(q, c)=q, \forall c \in \Sigma$. We call it the $O P$ Max-Automaton over $\Sigma, M$. For a max-automaton, each chain has a support. Since there is a chain ${ }^{\#}[s]^{\#}$ for any string $s$ compatible with $M$, a string is accepted by $\mathcal{A}(\Sigma, M)$ iff it is compatible with $M$. If $M$ is complete, each string is accepted by $\mathcal{A}(\Sigma, M)$, which defines the universal language $\Sigma^{*}$ by assigning to any string the (unique) structure compatible with the OPM. With $M_{\text {call }}$ of Fig. 1 , if we take e.g. the string ret call han, it is accepted by the max-automaton with structure \#[[ret $]$ call $[$ han $]] \#$.

In conclusion, given an OP alphabet, the OPM $M$ assigns a unique structure to any compatible string in $\Sigma^{*}$; unlike VPL, such a structure is not visible in the string, and must be built by means of a non-trivial parsing algorithm. An OPA defined on the OP alphabet selects an appropriate subset within the "universe" of strings compatible with $M$. For a more complete description of the OPL family and of its relations with other CFL we refer the reader to [43].

\subsection{Operator Precedence $\omega$-Languages}

All definitions regarding OPL are extended to infinite words in the usual way, but with a few distinctions. Given an OP alphabet $(\Sigma, M)$, an $\omega$-word $w \in \Sigma^{\omega}$ is compatible with $M$ if every prefix of $w$ is compatible with $M$. OP $\omega$-words are not terminated by the delimiter \#. An $\omega$-word may contain never-ending chains of the form $c_{0} \lessdot c_{1} \doteq c_{2} \doteq \cdots$, where the $\lessdot$ relation between $c_{0}$ and $c_{1}$ is never closed by a corresponding $\gg$. Such chains are called open chains and 
may be simple or composed. A composed open chain may contain both open and closed subchains. Of course, a closed chain cannot contain an open one. A terminal symbol $a \in \Sigma$ is pending if it is part of the body of an open chain and of no closed chains.

OPA classes accepting the whole class of $\omega$ OPL can be defined by augmenting Definition 1 with Büchi or Muller acceptance conditions [42]. In this paper, we only consider the former. The semantics of configurations, moves and infinite runs are defined as for finite OPA. For the acceptance condition, let $\rho$ be a run on an $\omega$-word $w$. Define

$$
\operatorname{Inf}(\rho)=\left\{q \in Q \mid \text { there exist infinitely many positions } i \text { s.t. }\left\langle\beta_{i}, q, x_{i}\right\rangle \in \rho\right\}
$$

as the set of states that occur infinitely often in $\rho . \rho$ is successful iff there exists a state $q_{f} \in F$ such that $q_{f} \in \operatorname{Inf}(\rho)$. An $\omega$ OPBA $\mathcal{A}$ accepts $w \in \Sigma^{\omega}$ iff there is a successful run of $\mathcal{A}$ on $w$. The $\omega$-language recognized by $\mathcal{A}$ is $L(\mathcal{A})=\left\{w \in \Sigma^{\omega} \mid\right.$ $\mathcal{A}$ accepts $w\}$. Unlike OPA, $\omega$ OPBA do not require the stack to be empty for word acceptance: when reading an open chain, the stack symbol pushed when the first character of the body of its underlying simple chain is read remains into the stack forever; it is at most updated by shift moves.

The most important closure properties of OPL are preserved by $\omega$ OPL, which form a Boolean algebra and are closed under concatenation of an OPL with an $\omega \mathrm{OPL}[42]$. The equivalence between deterministic and nondeterministic automata is lost in the infinite case, which is unsurprising, since it also happens for regular $\omega$-languages and $\omega \mathrm{VPL}$.

\subsection{Modeling Programs with OPA}

For readers not familiar with OPL, we show how OPA can naturally model programming languages such as Java and $\mathrm{C}++$. Given a set $A P$ of atomic propositions describing events and states of the program, we use $\left(\mathcal{P}(A P), M_{A P}\right)$ as the OP alphabet. For convenience, we consider a partitioning of $A P$ into a set of standard propositional labels (in round font), and structural labels (SL, in bold). SL define the OP structure of the word: $M_{A P}$ is only defined for subsets of $A P$ containing exactly one $\mathrm{SL}$, so that given two SL $\mathbf{l}_{1}, \mathbf{l}_{2}$, for any $a, a^{\prime}, b, b^{\prime} \in \mathcal{P}(A P)$ s.t. $\mathbf{l}_{1} \in a, a^{\prime}$ and $\mathbf{l}_{2} \in b, b^{\prime}$ we have $M_{A P}(a, b)=M_{A P}\left(a^{\prime}, b^{\prime}\right)$. Hence, we define an OPM on the entire $\mathcal{P}(A P)$ by only giving the relations between SL, as we did for $M_{\text {call }}$. Figure 2 shows how to model a procedural program with an OPA. The OPA simulates the program's behavior with respect to the stack, by expressing its execution traces with four event kinds: call (resp. ret) marks a procedure call (resp. return), han the installation of an exception handler by a try statement, and exc an exception being raised. OPM $M_{\text {call }}$ defines the context-free structure of the word, which is strictly linked with the programming language semantics: the $\lessdot \mathrm{PR}$ causes nesting (e.g., calls can be nested into other calls), and the $\doteq$ $\mathrm{PR}$ implies a one-to-one relation, e.g. between a call and the ret of the same function, and a han and the exc it catches. Each OPA state represents a line in the source code. First, procedure $\mathrm{p}_{A}$ is called by the program loader $(\mathrm{M} 0)$, 


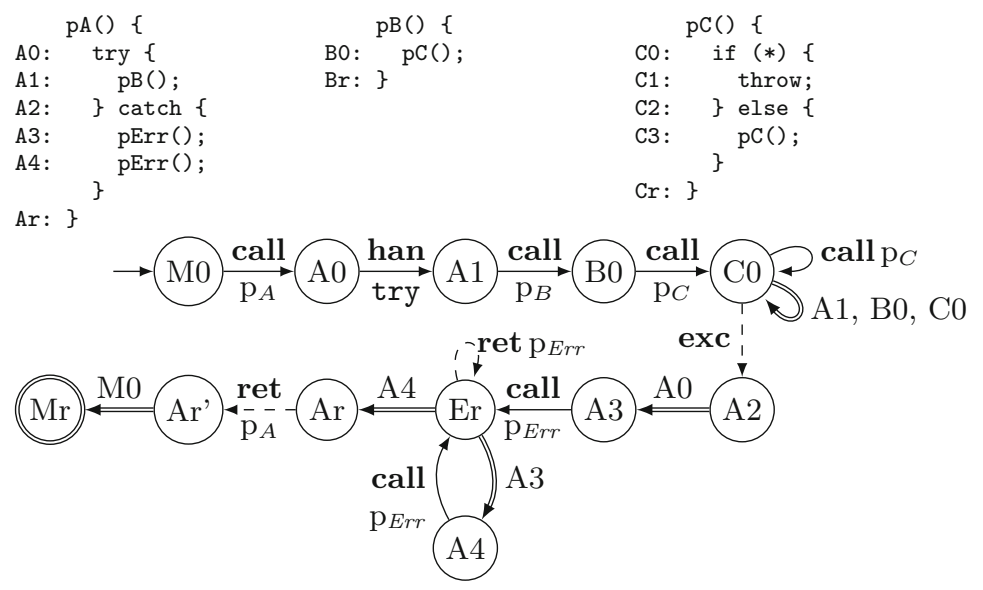

Fig. 2. Example procedural program (top) and the derived OPA (bottom). ' $*$ ' implies a non-deterministic choice. Push, shift, pop moves are shown by, resp., solid, dashed and double arrows.

and $\left[\left\{\right.\right.$ call, $\left.\left.\mathrm{p}_{A}\right\}, \mathrm{M} 0\right]$ is pushed onto the stack, to track the program state before the call. Then, the try statement at line $\mathrm{A} 0$ of $\mathrm{p}_{A}$ installs a handler. All subsequent calls to $\mathrm{p}_{B}$ and $\mathrm{p}_{C}$ push new stack symbols on top of the one pushed with han. $\mathrm{p}_{C}$ may only call itself recursively, or throw an exception, but never return normally. This is reflected by exc being the only transition leading from state $\mathrm{C} 0$ to the accepting state $\mathrm{Mr}$, and $\mathrm{p}_{B}$ and $\mathrm{p}_{C}$ having no way to a normal ret. The OPA has a look-ahead of one input symbol, so when it encounters exc, it must pop all symbols in the stack, corresponding to active function frames, until it finds the one with han in it, which cannot be popped because han $\doteq$ exc. Notice that such behavior cannot be modeled by Visibly Pushdown Automata or Nested Word Automata, because they need to read an input symbol for each pop move. Thus, han protects the parent function from the exception. Since the state contained in han's stack symbol is A0, the execution resumes in the catch clause of $\mathrm{p}_{A} \cdot \mathrm{p}_{A}$ then calls twice the error-handling function $\mathrm{p}_{E r r}$, which ends regularly both times, and returns. The string of Fig. 1 is accepted by this OPA.

In this example, we only model the stack behavior for simplicity, but other statements, such as assignments, and other behaviors, such as continuations, could be modeled by a different choice of the OPA and OPM, and other aspects of the program's state by appropriate abstractions [38].

\section{POTL: Syntax and Semantics}

Given a finite set of atomic propositions $A P$, the syntax of POTL follows:

$$
\begin{aligned}
\varphi::=\mathrm{a}|\neg \varphi| \varphi \vee \varphi\left|\bigcirc^{t} \varphi\right| \ominus^{t} \varphi\left|\chi_{F}^{t} \varphi\right| \chi_{P}^{t} \varphi\left|\varphi \mathcal{U}_{\chi}^{t} \varphi\right| \varphi \mathcal{S}_{\chi}^{t} \varphi \\
\quad\left|\bigcirc_{H}^{t} \varphi\right| \Theta_{H}^{t} \varphi\left|\varphi \mathcal{U}_{H}^{t} \varphi\right| \varphi \mathcal{S}_{H}^{t} \varphi
\end{aligned}
$$




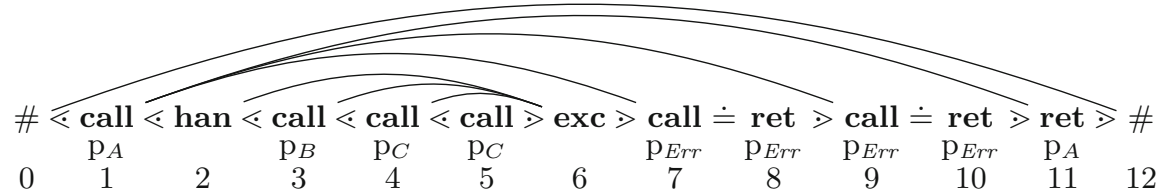

Fig. 3. The string of Fig. 1 as an OP word. Chains are shown by edges joining their contexts. Standard atomic propositions are shown below SL: $\mathrm{p}_{l}$ means a call or a ret is related to procedure $\mathrm{p}_{l}$. First, procedure $\mathrm{p}_{A}$ is called (pos. 1), and it installs a handler in pos. 2 . Then, three procedures are called, and one $\left(\mathrm{p}_{C}\right)$ throws an exception, which is caught by the handler. Two more functions are called and, finally, $\mathrm{p}_{A}$ returns.

where a $\in A P$, and $t \in\{d, u\}$.

The semantics of POTL is based on the word structure-also called OP word for short- $\left(U, M_{A P}, P\right)$, where $U=\{0,1, \ldots, n, n+1\}$, with $n \in \mathbb{N}$ is a set of word positions; $P: U \rightarrow \mathcal{P}(A P)$ is a function associating each position in $U$ with the set of atomic propositions holding in that position, with $P(0)=P(n+1)=\{\#\}$. Given two positions $i, j$ and a $\mathrm{PR} \pi$, we write $i \pi j$ to say $P(i) \pi P(j)$.

We define the chain relation $\chi \subseteq U \times U$ so that $\chi(i, j)$ holds between two positions $i, j$ iff $i<j-1$, and $i$ and $j$ are resp. the left and right contexts of the same chain. For composed chains, $\chi$ may not be one-to-one, but also one-to-many or many-to-one. Given $i, j \in U$, relation $\chi$ has the following properties:

1. It never crosses itself: if $\chi(i, j)$ and $\chi(h, k)$, for any $h, k \in U$, then we have $i<h<j \Longrightarrow k \leq j$ and $i<k<j \Longrightarrow i \leq h$.

2. If $\chi(i, j)$, then $i \lessdot i+1$ and $j-1>j$.

3. There exists at most one single position $h$, called leftmost context of $j$, s.t. $\chi(h, j)$ and $h \lessdot j$ or $h \doteq j$; for any $k$ s.t. $\chi(k, j)$ and $k \gg j$ we have $k>h$.

4. There exists at most one single position $h$, called rightmost context of $i$, s.t. $\chi(i, h)$ and $i>h$ or $i \doteq h$; for any $k$ s.t. $\chi(i, k)$ and $i \lessdot k$ we have $k<h$.

Property 4 says that when the chain relation is one-to-many, the contexts of the outermost chains are in the $\doteq$ or $>$ relation, while the inner ones are in the $\lessdot$ relation. Property 3 says that contexts of outermost many-to-one chains are in the $\doteq$ or $\lessdot$ relation, the inner ones being in the $>$ relation. In the $\mathrm{ST}$, the right context $j$ of a chain is at the same level as the left one $i$ when $i \doteq j$ (e.g., in Fig. 4, pos. 1 and 11), at a lower level when $i \lessdot j$ (e.g., pos. 1 with 7 , and 9), at a higher level if $i>j$ (e.g., pos. 3 and 4 with 6$)$.

The truth of POTL formulas is defined w.r.t. a single word position. Let $w$ be an OP word, and $\mathrm{a} \in A P$. Then, for any position $i \in U$ of $w$, we have $(w, i) \models$ a if a $\in P(i)$. Operators such as $\wedge$ and $\neg$ have the usual semantics from propositional logic. Next, while giving the formal semantics of POTL operators, we illustrate it by showing how it can be used to express properties on program execution traces, such as the one of Fig. 3. 
a) Next/Back Operators. The downward next and back operators $\bigcirc^{d}$ and $\Theta^{d}$ are like their LTL counterparts, except they are true only if the next (resp. current) position is at a lower or equal ST level than the current (resp. preceding) one. The upward next and back, $\bigcirc^{u}$ and $\ominus^{u}$, are symmetric. Formally, $(w, i) \models$ $\bigcirc^{d} \varphi$ iff $(w, i+1) \models \varphi$ and $i \lessdot(i+1)$ or $i \doteq(i+1)$, and $(w, i) \models \Theta^{d} \varphi$ iff $(w, i-1) \models \varphi$, and $(i-1) \lessdot i$ or $(i-1) \doteq i$. Substitute $\lessdot$ with $>$ to obtain the semantics for $\bigcirc^{u}$ and $\ominus^{u}$. E.g., we can write $\bigcirc^{d}$ call to say that the next position is an inner call (it holds in pos. 2, 3, 4 of Fig. 3), $\Theta^{d}$ call to say that the previous position is a call, and the current is the first of the body of a function (pos. 2, 4, 5), or the ret of an empty one (pos. 8, 10), and $\ominus^{u}$ call to say that the current position terminates an empty function frame (holds in $6,8,10)$. In pos. $2 \bigcirc^{d} \mathrm{p}_{B}$ holds, but $\bigcirc^{u} \mathrm{p}_{B}$ does not.

b) Chain Next/Back Operators. The chain next and back operators $\chi_{F}^{t}$ and $\chi_{P}^{t}, t \in\{d, u\}$, evaluate their argument respectively on future and past positions in the chain relation with the current one. The downward (resp. upward) variant only considers chains whose right context goes down (resp. up) in the ST. E.g., in pos. 1 of Fig. $3, \chi_{F}^{d} \mathrm{p}_{E r r}$ holds because $\chi(1,7)$ and $\chi(1,9)$, meaning that $\mathrm{p}_{A}$ calls $\mathrm{p}_{E r r}$ at least once. Formally, $(w, i) \models \chi_{F}^{d} \varphi$ iff there exists a position $j>i$ such that $\chi(i, j), i \lessdot j$ or $i \doteq j$, and $(w, j) \models \varphi$. $(w, i) \models \chi_{P}^{d} \varphi$ iff there exists a position $j<i$ such that $\chi(j, i), j \lessdot i$ or $j \doteq i$, and $(w, j) \models \varphi$. Replace $\lessdot$ with $>$ for the upward versions. In Fig. 3, $\chi_{F}^{u}$ exc is true in call positions whose procedure is terminated by an exception thrown by an inner procedure (e.g. pos. 3 and 4$) \cdot \chi_{P}^{u}$ call is true in exc statements that terminate at least one procedure other than the one raising it, such as the one in pos. 6. $\chi_{F}^{d}$ ret and $\chi_{F}^{u}$ ret hold in calls to non-empty procedures that terminate normally, and not due to an uncaught exception (e.g., pos. 1).

c) Until/Since Operators. POTL has two kinds of until and since operators. They express properties on paths, which are sequences of positions obtained by iterating the different kinds of next or back operators. In general, a path of length $n \in \mathbb{N}$ between $i, j \in U$ is a sequence of positions $i=i_{1}<i_{2}<\cdots<i_{n}=j$. The until operator on a set of paths $\Gamma$ is defined as follows: for any word $w$ and position $i \in U$, and for any two POTL formulas $\varphi$ and $\psi,(w, i) \models \varphi \mathcal{U}(\Gamma) \psi$ iff there exist a position $j \in U, j \geq i$, and a path $i_{1}<i_{2}<\cdots<i_{n}$ between $i$ and $j$ in $\Gamma$ such that $\left(w, i_{k}\right) \models \varphi$ for any $1 \leq k<n$, and $\left(w, i_{n}\right) \models \psi$. Since operators are defined symmetrically. Note that, depending on $\Gamma$, a path from $i$ to $j$ may not exist. We define until/since operators by associating them with different sets of paths. 
The summary until $\psi \mathcal{U}_{\chi}^{t} \theta$ (resp. since $\psi \mathcal{S}_{\chi}^{t} \theta$ ) operator is obtained by inductively applying the $\bigcirc^{t}$ and $\chi_{F}^{t}$ (resp. $\ominus^{t}$ and $\chi_{P}^{t}$ ) operators. It holds in a position in which either $\theta$ holds, or $\psi$ holds together with $\bigcirc^{t}\left(\psi \mathcal{U}_{\chi}^{t} \theta\right)\left(\operatorname{resp} . \ominus^{t}\left(\psi \mathcal{S}_{\chi}^{t} \theta\right)\right)$ or $\chi_{F}^{t}\left(\psi \mathcal{U}_{\chi}^{t} \theta\right)$ (resp. $\left.\chi_{P}^{t}\left(\psi \mathcal{S}_{\chi}^{t} \theta\right)\right)$. It is an until operator on paths that can move not only between consecutive positions, but also between contexts of a chain, skipping its body. With the OPM of Fig. 1, this means skipping function bodies. The downward variants can move between positions at the same level in the ST (i.e., in the same simple chain body), or down in the nested chain structure. The upward ones remain at the same level, or move to higher levels of the ST.

Formula $\top \mathcal{U}_{\chi}^{u}$ exc is true in positions contained in the frame of a function that is terminated by an exception. It is true in pos. 3 of Fig. 3 because of path $3-6$, and false in pos. 1, because no path can enter the chain whose contexts are pos. 1 and 11. Formula $\top \mathcal{U}_{\chi}^{d}$ exc is true in call positions whose function frame contains excs, but that are not necessarily terminated by one of them, such as the one in pos. 1 (with path 1-2-6).

We define Downward Summary Paths (DSP) as follows. Given an OP word $w$, and two positions $i \leq j$ in $w$, the DSP between $i$ and $j$, if it exists, is a sequence of positions $i=i_{1}<i_{2}<\cdots<i_{n}=j$ such that, for each $1 \leq p<n$,

$$
i_{p+1}= \begin{cases}k & \text { if } k=\max \left\{h \mid h \leq j \wedge \chi\left(i_{p}, h\right) \wedge\left(i_{p} \lessdot h \vee i_{p} \doteq h\right)\right\} \text { exists; } \\ i_{p}+1 & \text { otherwise, if } i_{p} \lessdot\left(i_{p}+1\right) \text { or } i_{p} \doteq\left(i_{p}+1\right)\end{cases}
$$

The Downward Summary (DS) until and since operators $\mathcal{U}_{\chi}^{d}$ and $\mathcal{S}_{\chi}^{d}$ use as $\Gamma$ the set of DSP starting in the position in which they are evaluated. The definition for the upward counterparts is, again, obtained by substituting $\lessdot$ with $>$. In Fig. 3, call $\mathcal{U}_{\chi}^{d}\left(\right.$ ret $\left.\wedge \mathrm{p}_{E r r}\right)$ holds in pos. 1 because of path 1-7-8 and 1-9-10, $\left(\right.$ call $\vee$ exc) $\mathcal{S}_{\chi}^{u} \mathrm{p}_{B}$ in pos. 7 because of path $3-6-7$, and (call $\vee$ exc) $\mathcal{U}_{\chi}^{u}$ ret in 3 because of path $3-6-7-8$.

d) Hierarchical Operators. A single position may be the left or right context of multiple chains. The operators seen so far cannot keep this fact into account, since they "forget" about a left context when they jump to the right one. Thus, we introduce the hierarchical next and back operators. The upward hierarchical next (resp. back), $\bigcirc_{H}^{u} \psi$ (resp. $\ominus_{H}^{u} \psi$ ), is true iff the current position $j$ is the right context of a chain whose left context is $i$, and $\psi$ holds in the next (resp. previous) pos. $j^{\prime}$ that is the right context of $i$, with $i \lessdot j, j^{\prime}$. So, $\bigcirc_{H}^{u} \mathrm{p}_{E r r}$ holds in pos. 7 of Fig. 3 because $\mathrm{p}_{E r r}$ holds in 9 , and $\ominus_{H}^{u} \mathrm{p}_{E r r}$ in 9 because $\mathrm{p}_{E r r}$ holds in 7 . In the $\mathrm{ST}, \bigcirc_{H}^{u}$ goes $u p$ between calls to $\mathrm{p}_{E r r}$, while $\ominus_{H}^{u}$ goes down. Their downward counterparts behave symmetrically, and consider multiple inner chains sharing their right context. They are formally defined as:

- $(w, i) \models \bigcirc_{H}^{u} \varphi$ iff there exist a position $h<i$ s.t. $\chi(h, i)$ and $h \lessdot i$ and a position $j=\min \{k \mid i<k \wedge \chi(h, k) \wedge h \lessdot k\}$ and $(w, j) \models \varphi$;

- $(w, i) \models \Theta_{H}^{u} \varphi$ iff there exist a position $h<i$ s.t. $\chi(h, i)$ and $h \lessdot i$ and a position $j=\max \{k \mid k<i \wedge \chi(h, k) \wedge h \lessdot k\}$ and $(w, j) \models \varphi$;

- $(w, i) \models \bigcirc_{H}^{d} \varphi$ iff there exist a position $h>i$ s.t. $\chi(i, h)$ and $i>h$ and a position $j=\min \{k \mid i<k \wedge \chi(k, h) \wedge k>h\}$ and $(w, j) \models \varphi$; 
- $(w, i) \models \Theta_{H}^{d} \varphi$ iff there exist a position $h>i$ s.t. $\chi(i, h)$ and $i>h$ and a position $j=\max \{k \mid k<i \wedge \chi(k, h) \wedge k>h\}$ and $(w, j) \models \varphi$.

In the ST of Fig. 4, $\bigcirc_{H}^{d}$ and $\ominus_{H}^{d}$ go down and up among calls terminated by the same exc. For example, in pos. $3 \bigcirc_{H}^{d} \mathrm{p}_{C}$ holds, because both pos. 3 and 4 are in the chain relation with 6 . Similarly, in pos. $4 \ominus_{H}^{d} \mathrm{p}_{B}$ holds. Note that these operators do not consider leftmost/rightmost contexts, so $\bigcirc_{H}^{u}$ ret is false in pos. 9 , as call $\doteq$ ret, and pos. 11 is the rightmost context of pos. 1 .

The hierarchical until and since operators are defined by iterating these next and back operators. The upward hierarchical path (UHP) between $i$ and $j$ is a sequence of positions $i=i_{1}<i_{2}<\cdots<i_{n}=j$ such that there exists a position $h<i$ such that for each $1 \leq p \leq n$ we have $\chi\left(h, i_{p}\right)$ and $h \lessdot i_{p}$, and for each $1 \leq q<n$ there exists no position $k$ such that $i_{q}<k<i_{q+1}$ and $\chi(h, k)$. The until and since operators based on the set of UHP starting in the position in which they are evaluated are denoted as $\mathcal{U}_{H}^{u}$ and $\mathcal{S}_{H}^{u}$. E.g., call $\mathcal{U}_{H}^{u}$ p Err $_{\text {holds }}$ in pos. 7 because of the singleton path 7 and path $7-9$, and call $\mathcal{S}_{H}^{u} \mathrm{p}_{E r r}$ in pos. 9 because of paths 9 and 7-9.

The downward hierarchical path (DHP) between $i$ and $j$ is a sequence of positions $i=i_{1}<i_{2}<\cdots<i_{n}=j$ such that there exists a position $h>j$ such that for each $1 \leq p \leq n$ we have $\chi\left(i_{p}, h\right)$ and $i_{p}>h$, and for each $1 \leq q<n$ there exists no position $k$ such that $i_{q}<k<i_{q+1}$ and $\chi(k, h)$. The until and since operators based on the set of DHP starting in the position in which they are evaluated are denoted as $\mathcal{U}_{H}^{d}$ and $\mathcal{S}_{H}^{d}$. In Fig. 3, call $\mathcal{U}_{H}^{d}$ p $\mathrm{p}_{C}$ holds in pos. 3, and call $\mathcal{S}_{H}^{d} \mathrm{p}_{B}$ in pos. 4 , both because of path $3-4$.

The POTL until and since operators enjoy expansion laws similar to those of LTL. Here we give those for two until operators, those for their since and downward counterparts being symmetric.

$$
\begin{aligned}
\varphi \mathcal{U}_{\chi}^{t} \psi & \equiv \psi \vee\left(\varphi \wedge\left(\bigcirc^{t}\left(\varphi \mathcal{U}_{\chi}^{t} \psi\right) \vee \chi_{F}^{t}\left(\varphi \mathcal{U}_{\chi}^{t} \psi\right)\right)\right) \\
\varphi \mathcal{U}_{H}^{u} \psi & \equiv\left(\psi \wedge \chi_{P}^{d} \top \wedge \neg \chi_{P}^{u} \top\right) \vee\left(\varphi \wedge \bigcirc_{H}^{u}\left(\varphi \mathcal{U}_{H}^{u} \psi\right)\right)
\end{aligned}
$$

\subsection{Expressiveness of POTL}

We first define some derived operators. For $t \in\{d, u\}$, we define the downward/upward summary eventually as $\diamond^{t} \varphi:=\top \mathcal{U}_{\chi}^{t} \varphi$, and the downward/upward summary globally as $\square^{t} \varphi:=\neg \diamond^{t}(\neg \varphi) . \diamond^{u} \varphi$ and $\square^{u} \varphi$ resp. say that $\varphi$ holds in one or all positions in the path from the current position to the root of the ST. $\diamond^{d} \varphi$ says that $\varphi$ holds in at least one position in the current subtree, and $\square^{d} \varphi$ in all of them. E.g., if $\square^{d}\left(\neg \mathrm{p}_{A}\right)$ holds in a call, it means that $\mathrm{p}_{A}$ never holds in its whole function body, which is the subtree rooted next to the call.

In the technical report, we prove

Theorem 1 ([23]). POTL $=$ FOL with one free variable on OP words.

Equivalence to FOL on the relevant algebraic structure is a desirable feature of linear-time temporal logics, and it was proved for LTL [39] and NWTL [2]. It 
is in some sense a theoretical assurance of the sufficient expressive power of the logic. Moreover, NWTL $\subset$ OPTL was proved in [22], and OPTL $\subseteq$ POTL comes from Theorem 1 and the semantics of OPTL being expressible in FOL. In [23], we also prove that there exist POTL formulas not expressible in OPTL. Thus, we can claim CaRet $[6] \subseteq$ NWTL $\subset$ OPTL $\subset$ POTL. One of such formulas is $\diamond^{d} \mathrm{p}_{A}$ which, evaluated e.g. on a han position with a matched exc, states that $\mathrm{p}_{A}$ holds in one of the positions in the same subtree.

More importantly, POTL can express many useful requirements of procedural programs. To emphasize the potential practical applications in automatic verification, we supply a few examples of typical program properties expressed as POTL formulas, not all of them being expressible in the other above languages.

The LTL globally can be written as $\square \psi:=\neg \diamond^{u}\left(\diamond^{d} \neg \psi\right)$. The two nested eventually operators enumerate all future positions by going up and then down in any direction in the syntax tree: when negated, this means $\neg \psi$ may never hold. POTL can express Hoare-style pre/postconditions with formulae such as $\square\left(\right.$ call $\wedge \rho \Longrightarrow \chi_{F}^{d}($ ret $\left.\wedge \theta)\right)$, where $\rho$ is the precondition, and $\theta$ is the postcondition.

Unlike NWTL, POTL can easily express properties related to exception handling and interrupt management [43]. E.g., the shortcut CallThr $(\psi):=\bigcirc^{u}(\mathbf{e x c} \wedge$ $\psi) \vee \chi_{F}^{u}(\mathbf{e x c} \wedge \psi)$, evaluated in a call, states that the procedure currently started is terminated by a exc in which $\psi$ holds. So, $\square$ (call $\wedge \rho \wedge \operatorname{CallThr}(\top) \Longrightarrow$ CallThr $(\theta))$ means that if precondition $\rho$ holds when a procedure is called, then postcondition $\theta$ must hold if that procedure is terminated by an exception. In object oriented programming languages, if $\rho \equiv \theta$ is a class invariant asserting that a class instance's state is valid, this formula expresses weak exception safety [1], and strong exception safety if $\rho$ and $\theta$ express particular states of the class instance. The no-throw guarantee can be stated with $\square\left(\right.$ call $\left.\wedge \mathrm{p}_{A} \Longrightarrow \neg \operatorname{CallThr}(\top)\right)$, meaning procedure $\mathrm{p}_{A}$ is never interrupted by an exception.

Stack inspection [29,37], i.e. properties regarding the sequence of procedures active in the program's stack at a certain point of its execution, is an important class of requirements that can be expressed with $\operatorname{shortcut} \operatorname{Scall}(\varphi, \psi):=$ $($ call $\Longrightarrow \varphi) \mathcal{S}_{\chi}^{d}($ call $\wedge \psi)$, which subsumes the call since of CaRet, as it also works with exceptions. E.g., $\square\left(\left(\right.\right.$ call $\left.\left.\wedge \mathrm{p}_{B} \wedge \operatorname{Scall}\left(\top, \mathrm{p}_{A}\right)\right) \Longrightarrow \operatorname{CallThr}(\top)\right)$ means that whenever $\mathrm{p}_{B}$ is executed and at least one instance of $\mathrm{p}_{A}$ is on the stack, $\mathrm{p}_{B}$ is terminated by an exception. The OPA of Fig. 2 satisfies this formula, because $\mathrm{p}_{B}$ is called by $\mathrm{p}_{A}$, and $\mathrm{p}_{C}$ throws.

\section{Model Checking}

Given an $\mathrm{OP}$ alphabet $\left(\mathcal{P}(A P), M_{A P}\right)$, where $A P$ is a finite set of atomic propositions, and a POTL formula $\varphi$, we build an OPA $\mathcal{A}_{\varphi}=\left(\mathcal{P}(A P), M_{A P}, Q, I, F, \delta\right)$ that accepts models of $\varphi$. The construction of $\mathcal{A}_{\varphi}$ resembles the classical one for LTL and the ones for NWTL and OPTL, diverging from them significantly when dealing with temporal obligations that involve positions in the chain relation.

We first introduce $C l(\varphi)$, the closure of $\varphi$, containing all subformulas of $\varphi$, and some auxiliary operators. The latter are needed to model-check chain 
next and back operators. For any $\operatorname{PR} \pi \in\{\lessdot, \doteq, \gg\}$, we define them as follows: $(w, i)=\chi_{F}^{\pi} \varphi$ iff there exists $j>i$ such that $\chi(i, j), i \pi j$, and $(w, j) \models \varphi$; $(w, i) \models \chi_{P}^{\pi} \varphi$ iff there exists $j<i$ such that $\chi(j, i), j \pi i$, and $(w, j) \models \varphi$.

$C l(\varphi)$ is the smallest set such that, for $t \in\{d, u\}$ :

1. $\varphi \in C l(\varphi)$,

2. $A P \subseteq C l(\varphi)$,

3. if $\psi \in C l(\varphi)$ and $\psi \neq \neg \theta$, then $\neg \psi \in C l(\varphi)$ (we identify $\neg \neg \psi$ with $\psi$ );

4. if $\neg \psi \in C l(\varphi)$, then $\psi \in C l(\varphi)$;

5. if any of $\psi \wedge \theta$ or $\psi \vee \theta$ is in $C l(\varphi)$, then $\psi, \theta \in C l(\varphi)$;

6. if any of $\bigcirc^{t} \psi, \Theta^{t} \psi, \chi_{F}^{t} \psi$, or $\chi_{P}^{t} \psi$ is in $C l(\varphi)$, then $\psi \in C l(\varphi)$;

7. if $\chi_{F}^{d} \psi\left(\operatorname{resp} . \chi_{F}^{u} \psi\right)$ is in $C l(\varphi)$, then $\chi_{F}^{\leftarrow} \psi\left(\operatorname{resp} . \chi_{F}^{\vec{p}} \psi\right), \chi_{\bar{F}}^{\dot{\bar{F}}} \psi, \chi_{L}$ are in it;

8. if $\chi_{P}^{d} \psi\left(\right.$ resp. $\left.\chi_{P}^{u} \psi\right)$ is in $C l(\varphi)$, then $\chi_{P}^{\leftarrow} \psi\left(\right.$ resp. $\left.\chi_{P}^{\vec{P}} \psi\right), \chi_{P}^{\dot{\bar{P}}} \psi$ are in it;

9. if any of $\psi \mathcal{U}_{\chi}^{t} \theta, \psi \mathcal{S}_{\chi}^{t} \theta, \psi \mathcal{U}_{H}^{t} \theta$, or $\psi \mathcal{S}_{H}^{t} \theta$ is in $C l(\varphi)$, then $\psi, \theta \in C l(\varphi)$;

10. if $\psi \mathcal{U}_{\chi}^{t} \theta \in C l(\varphi)$, then $\bigcirc^{t}\left(\psi \mathcal{U}_{\chi}^{t} \theta\right), \chi_{F}^{t}\left(\psi \mathcal{U}_{\chi}^{t} \theta\right) \in C l(\varphi)$ (since is symmetric).

The set $\operatorname{Atoms}(\varphi)$ contains all consistent subsets of $C l(\varphi)$, i.e. all $\Phi \subseteq C l(\varphi)$ s.t.

- for every $\psi \in C l(\varphi), \psi \in \Phi$ iff $\neg \psi \notin \Phi$;

$-\psi \wedge \theta \in \Phi$, iff $\psi \in \Phi$ and $\theta \in \Phi$

- $\psi \vee \theta \in \Phi$, iff $\psi \in \Phi$ or $\theta \in \Phi$, or both.

The consistency constraints on Atoms $(\varphi)$ will be augmented incrementally in the following, for each operator.

The set of states of $\mathcal{A}_{\varphi}$ is $Q=\operatorname{Atoms}(\varphi)^{2}$, and its elements, which we denote with Greek capital letters, are of the form $\Phi=\left(\Phi_{c}, \Phi_{p}\right)$, where $\Phi_{c}$ is the set of formulas that hold in the current position, and $\Phi_{p}$ is the set of temporal obligations. The latter keep track of arguments of temporal operators that must be satisfied after a chain body, skipping it. The way they do so depends on the transition relation $\delta$, which we also define incrementally. Each automaton state is associated to word positions. So, for $(\Phi, a, \Psi) \in \delta_{\text {push } / \text { shift }}$, with $\Phi \in \operatorname{Atoms}(\varphi)^{2}$ and $a \in \mathcal{P}(A P)$, we have $\Phi_{c} \cap A P=a$ (by $\Phi_{c} \cap A P$ we mean the set of atomic propositions in $\left.\Phi_{c}\right)$. Pop moves do not read input symbols, and the automaton remains at the same position when performing them: for any $(\Phi, \Theta, \Psi) \in \delta_{\text {pop }}$ we impose $\Phi_{c}=\Psi_{c}$. The initial set $I$ contains states of the form $\left(\Phi_{c}, \Phi_{p}\right)$, with $\varphi \in \Phi_{c}$, and the final set $F$ states of the form $\left(\Psi_{c}, \Psi_{p}\right)$, s.t. $\Psi_{c} \cap A P=\{\#\}$ and $\Psi_{c}$ contains no future operators. We extend the construction to the most important operators, leaving the others and correctness proofs to [21].

Next/Back Operators. Let $(\Phi, a, \Psi) \in \delta_{\text {shift }} \cup \delta_{\text {push }}$, with $\Phi, \Psi \in \operatorname{Atoms}(\varphi)^{2}$, $a \in \mathcal{P}(A P)$, and let $b=\Psi_{c} \cap A P$ : we have $\bigcirc^{d} \psi \in \Phi_{c}$ iff $\psi \in \Psi_{c}$ and either $a \lessdot b$ or $a \doteq b$. The constraints introduced for the $\ominus^{d}$ operator are symmetric, and for their upward counterparts it suffices to replace $\lessdot$ with $>$.

If $\chi_{F}^{d} \psi \in C l(\varphi)$, for each $\Phi \in \operatorname{Atoms}(\varphi)^{2}$ we impose that $\chi_{F}^{d} \psi \in \Phi_{c}$ iff $\chi_{F}^{\leftarrow} \psi \in \Phi_{c}$ or $\chi_{\bar{F}}^{\dot{\bar{F}}} \psi \in \Phi_{c}$. Analogous rules are defined for the upward and past chain operators. The auxiliary symbol $\chi_{L}$ forces the current position to be the first one of a chain body. Let the current state of the OPA be $\Phi \in \operatorname{Atoms}(\varphi)^{2}$ : 


\begin{tabular}{|c|c|c|c|c|c|c|c|}
\hline & input & state & & stac & & PR & move \\
\hline 1 & call han exc ret \# & $\begin{array}{l}\Phi_{c}^{0}=\left\{\text { call }, \chi_{F}^{d} \text { ret }, \chi_{\bar{F}} \text { ret }\right\} \\
\Phi_{p}^{0}=\left\{\chi_{L}\right\}\end{array}$ & & & $\perp$ & $\# \lessdot$ call & push \\
\hline 2 & han exc ret \# & $\Phi^{1}=\left(\{\right.$ han $\},\left\{\chi \dot{\overline{\bar{F}}}\right.$ ret, $\left.\left.\chi_{L}\right\}\right)$ & & {$\left[\right.$ call, $\left.\Phi^{0}\right]$} & & call $\lessdot$ han & push \\
\hline 3 & exc ret \# & $\Phi^{2}=(\{\mathbf{e x c}\}, \emptyset)$ & {$\left[\right.$ han, $\Phi^{1}$} & {$\left[\right.$ call, $\left.\Phi^{0}\right]$} & $\perp$ & han $\doteq$ exc & shift \\
\hline 4 & ret \# & $\Phi^{3}=(\{\boldsymbol{r e t}\}, \emptyset)$ & {$\left[\mathbf{e x c}, \Phi^{1}\right.$} & {$\left[\right.$ call, $\left.\Phi^{0}\right]$} & $\perp$ & exc $>$ ret & pop \\
\hline 5 & ret \# & $\Phi^{4}=(\{$ ret $\},\{\chi \dot{\overline{\bar{F}}}$ ret $\})$ & & {$\left[\right.$ call, $\left.\Phi^{0}\right]$} & $\perp$ & call $\doteq$ ret & shift \\
\hline 6 & \# & $\Phi^{5}=(\{\#\}, \emptyset)$ & & {$\left[\operatorname{ret}, \Phi^{0}\right]$} & $\perp$ & ret $>\#$ & pop \\
\hline 7 & $\#$ & $\Phi^{5}=(\{\#\}, \emptyset)$ & & & $\perp$ & - & - \\
\hline
\end{tabular}

Fig. 5. Example accepting run of the automaton for $\chi_{F}^{d}$ ret.

$\chi_{L} \in \Phi_{p}$ iff the next transition (i.e. the one reading the current position) is a push. Formally, if $(\Phi, a, \Psi) \in \delta_{\text {shift }}$ or $(\Phi, \Theta, \Psi) \in \delta_{\text {pop }}$, for any $\Phi, \Theta, \Psi$ and $a$, then $\chi_{L} \notin \Phi_{p}$. If $(\Phi, a, \Psi) \in \delta_{p u s h}$, then $\chi_{L} \in \Phi_{p}$. For any initial state $\left(\Phi_{c}, \Phi_{p}\right) \in I$, we have $\chi_{L} \in \Phi_{p}$ iff $\# \notin \Phi_{c}$.

If $\chi \dot{\bar{F}} \psi \in C l(\varphi)$, its satisfaction is ensured by the following constraints on $\delta$ :

1. Let $(\Phi, a, \Psi) \in \delta_{\text {push } / \text { shift }}$ : then $\chi_{\overline{\bar{F}}}^{\dot{\bar{F}}} \psi \in \Phi_{c}$ iff $\chi_{\overline{\bar{F}}}^{\dot{\bar{F}}} \psi, \chi_{L} \in \Psi_{p}$;

2. let $(\Phi, \Theta, \Psi) \in \delta_{\text {pop }}$ : then $\chi_{\overline{\bar{F}}}^{\dot{\bar{\nu}}} \notin \Phi_{p}$, and $\chi_{\overline{\bar{F}}} \psi \in \Theta_{p}$ iff $\chi \dot{\overline{\bar{F}}} \psi \in \Psi_{p}$;

3. let $(\Phi, a, \Psi) \in \delta_{\text {shift }}$ : then $\chi_{\bar{F}}^{\overline{\bar{F}}} \psi \in \Phi_{p}$ iff $\psi \in \Phi_{c}$.

If $\chi_{F}^{\leftarrow} \psi \in C l(\varphi), \chi_{F}^{\leftarrow} \psi$ is allowed in the pending part of initial states, and we add the following constraints:

4. Let $(\Phi, a, \Psi) \in \delta_{\text {push } / \text { shift }}$ : then $\chi_{F}^{\leftarrow} \psi \in \Phi_{c}$ iff $\chi_{F}^{\leftarrow} \psi, \chi_{L} \in \Psi_{p}$;

5. let $(\Phi, \Theta, \Psi) \in \delta_{p o p}$ : then $\chi_{F}^{\leftarrow} \psi \in \Theta_{p}$ iff $\chi_{L} \in \Psi_{p}$, and either $\chi_{F}^{\leftarrow} \psi \in \Psi_{p}$ or $\psi \in \Phi_{c}$.

We illustrate how the construction works for $\chi_{\bar{F}}$ with the example of Fig. 5 . The OPA starts in state $\Phi^{0}$, with $\chi_{F}^{d}$ ret $\in \Phi_{c}^{0}$, and guesses that $\chi_{F}^{d}$ will be fulfilled by $\chi \dot{\bar{F}}$, so $\chi \dot{\bar{F}}$ ret $\in \Phi_{c}^{0}$. call is read by a push move, resulting in state $\Phi^{1}$. The OPA guesses the next move will be a push, so $\chi_{L} \in \Phi_{p}^{1}$. By rule 1 , we have $\chi_{\bar{F}}$ ret $\in \Phi_{p}^{1}$. The last guess is immediately verified by the next push (step $2-3)$. Thus, the pending obligation for $\chi \dot{\overline{\bar{F}}}$ ret is stored onto the stack in $\Phi^{1}$. The OPA, then, reads exc with a shift, and pops the stack symbol containing $\Phi^{1}$ (step 4-5). By rule 2, the temporal obligation is resumed in the next state $\Phi^{4}$, so $\chi \dot{\bar{F}}$ ret $\in \Phi_{p}^{4}$. Finally, ret is read by a shift which, by rule 3 , may occur only if ret $\in \Phi_{c}^{4}$. Rule 3 verifies the guess that $\chi_{\bar{F}}$ ret holds in $\Phi_{0}$, and fulfills the temporal obligation contained in $\Phi_{p}^{4}$, by preventing computations in which ret $\notin \Phi_{c}^{4}$ from continuing. Had the next transition been a pop (e.g. because there was no ret and call $>\#$ ), the run would have been blocked by rule 2 , preventing the OPA from reaching an accepting state, and from emptying the stack.

Summary Until and Since. The construction for these operators is based on their expansion laws. For any $\Phi \in \operatorname{Atoms}(\varphi)^{2}$, we have $\psi \mathcal{U}_{\chi}^{t} \theta \in \Phi_{c}$, with 
$t \in\{d, u\}$ being a direction, iff either: 1. $\theta \in \Phi_{c}, 2 . \bigcirc^{t}\left(\psi \mathcal{U}_{\chi}^{t} \theta\right), \psi \in \Phi_{c}$, or 3 . $\chi_{F}^{t}\left(\psi \mathcal{U}_{\chi}^{t} \theta\right), \psi \in \Phi_{c}$. The rules for since are symmetric.

Hierarchical Operators. For the hierarchical operators, we do not give an explicit OPA construction, but we rely on a translation into other POTL operands. For each hierarchical operator $\eta$ in $\varphi$, we add a propositional symbol $q_{(\eta)}$. The upward hierarchical operators consider the right contexts of chains sharing the same left context. To distinguish such positions, we define formula $\gamma_{L, \eta}:=\chi_{P}^{\lessdot}\left(\mathrm{q}_{(\eta)} \wedge \bigcirc\left(\square \neg \mathrm{q}_{(\eta)}\right) \wedge \ominus\left(\boxminus \neg \mathrm{q}_{(\eta)}\right)\right)$, where $\square$ and $\boxminus$ are as in Sect. 3.1. $\bigcirc$ and $\Theta$ are the LTL next and back operators, for which model checking can be done as for $\bigcirc^{d}$ and $\Theta^{d}$, but removing the restrictions on PR. $\gamma_{L, \eta}$, evaluated on a position $i$, asserts that $\mathrm{q}_{(\eta)}$ holds in the unique position $h$ such that $\chi(h, i)$ and $h \lessdot i$. Thus, $\mathrm{q}_{(\eta)}$ can be used to distinguish other positions $j$ such that $\chi(h, j)$ and $h \lessdot j$, as $\chi_{P}^{\leftarrow} \mathrm{q}_{(\eta)}$ holds in them. The translations for future upward hierarchical operators follow, the others being analogous.

$$
\begin{aligned}
\bigcirc_{H}^{u} \psi & :=\gamma_{L, \bigcirc_{H}^{u} \psi} \wedge \bigcirc\left(\left(\neg \chi_{P}^{\leftarrow} \mathrm{q}_{\left(\bigcirc_{H}^{u} \psi\right)}\right) \mathcal{U}_{\chi}^{u}\left(\chi_{P}^{\leftarrow} \mathrm{q}_{\left(\bigcirc_{H}^{u} \psi\right)} \wedge \psi\right)\right) \\
\psi \mathcal{U}_{H}^{u} \theta & :=\gamma_{L, \psi \mathcal{U}_{H}^{u} \theta} \wedge\left(\chi_{P}^{\leftarrow} \mathrm{q}_{\left(\psi \mathcal{U}_{H}^{u} \theta\right)} \Longrightarrow \psi\right) \mathcal{U}_{\chi}^{u}\left(\chi_{P}^{\leftarrow} \mathrm{q}_{\left(\psi \mathcal{U}_{H}^{u} \theta\right)} \wedge \theta\right)
\end{aligned}
$$

\subsection{Model Checking for $\omega$-Words}

To perform model checking of a POTL formula $\varphi$ on OP $\omega$-words, we build a generalized $\omega$ OPBA $\mathcal{A}_{\varphi}^{\omega}=\left(\mathcal{P}(A P), M_{A P}, Q_{\omega}, I, \mathbf{F}, \delta\right)$, where $Q_{\omega}=\operatorname{Atoms}(\varphi)^{2} \times$ $\mathcal{P}\left(C l_{\text {stack }}(\varphi)\right)$, which differs from the finite-word OPA only for the state set and the acceptance condition. As in [2], the generalized Büchi acceptance condition is a slight variation on the one shown in Sect. 2.1: $\mathbf{F}$ is the set of sets of Büchi final states, and an $\omega$-word is accepted iff at least one state from each one of the sets contained in $\mathbf{F}$ is visited infinitely often during the computation.

In finite words, the stack is empty at the end of every accepting computation, which implies the satisfaction of all temporal constraints tracked by the pending part of stack symbols. In $\omega$ OPBAs, the stack may never be empty, and symbols with a non-empty pending part may remain in it indefinitely, never enforcing the satisfaction of the respective formulas. To overcome this issue, we use $\operatorname{Atoms}(\varphi)^{2} \times \mathcal{P}\left(C l_{\text {stack }}(\varphi)\right)$, with $C l_{\text {stack }}(\varphi) \subseteq C l(\varphi)$, as the state set of the $\omega$ OPBA. Such states have the form $\Phi=\left(\Phi_{c}, \Phi_{p}, \Phi_{s}\right)$, where $\Phi_{c}$ and $\Phi_{p}$ have the same role as in the finite-word case, and $\Phi_{s}$ is the in-stack part of $\Phi$. All rules previously defined for $\Phi_{c}$ and $\Phi_{p}$ remain the same. $\Phi_{s}$ contains elements of $C l_{\text {stack }}(\varphi)$ contained in any symbol currently on the stack. $C l_{\text {stack }}(\varphi)$ contains formulas in $\mathrm{Cl}(\varphi)$ that use the stack to ensure the satisfaction of future temporal requirements, namely all $\chi_{F}^{\pi} \psi \in C l(\varphi)$, with $\pi \in\{\lessdot, \doteq, \gg\}$. Thus, pending temporal obligations are moved from the stack to the $\omega$ OPBA state, and they can be considered by the Büchi acceptance condition.

Suppose we want to model check $\chi \dot{\bar{F}} \psi$. Formula $\chi_{\bar{F}}^{\dot{\bar{F}}} \psi$ must be inserted in the in-stack part of the current state whenever a stack symbol containing it in its pending part is pushed. It must be kept in the in-stack part of the current state until the last stack symbol containing it in its pending part is popped, marking 


\begin{tabular}{|c|c|c|c|c|}
\hline & input & state & stack & PR \\
\hline & call call han exc ret ret $(\text { call })^{\omega}$ & $\begin{aligned} \Phi^{0}= & \left(\left\{\text { call }, \chi_{F}^{d} \text { ret }, \chi_{\bar{F}} \text { ret }\right\},\right. \\
& \left.\left\{\chi_{L}\right\}, \emptyset\right)\end{aligned}$ & $\perp$ & $\lessdot$ \\
\hline 2 & call han exc ret ret $(\text { call })^{\omega}$ & 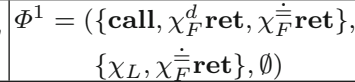 & {$\left[\right.$ call, $\left.\Phi^{0}\right] \perp$} & $\lessdot$ \\
\hline 3 & han exc ret ret $(\text { call })^{\omega}$ & $\begin{aligned} \Phi^{2}= & \left(\{\text { han }\},\left\{\chi_{L}, \chi \overline{\overline{\bar{F}}} \mathbf{r e t}\right\}\right. \\
& \{\chi \dot{\overline{\bar{F}}} \mathbf{r e t}\})\end{aligned}$ & {$\left[\right.$ call, $\left.\Phi^{1}\right]\left[\right.$ call, $\left.\Phi^{0}\right] \perp$} & $\lessdot$ \\
\hline 4 & exc ret ret $(\text { call })^{\omega}$ & $\Phi^{3}=(\{\mathbf{e x c}\}, \emptyset,\{\chi \dot{\bar{F}} \mathbf{r e t}\})$ & {$\left[\right.$ han,$\left.\Phi^{2}\right]\left[\right.$ call, $\left.\Phi^{1}\right]\left[\right.$ call,$\left.\Phi^{0}\right] \perp$} & $\doteq$ \\
\hline 5 & ret ret $(\text { call })^{\omega}$ & $\Phi^{4}=(\{$ ret $\}, \emptyset,\{\chi \overline{\bar{F}}$ ret $\})$ & {$\left[\mathbf{e x c}, \Phi^{2}\right]\left[\right.$ call,$\left.\Phi^{1}\right]\left[\right.$ call,$\left.\Phi^{0}\right] \perp$} & $\rightarrow$ \\
\hline 6 & ret ret $(\text { call })^{\omega}$ & $\begin{aligned} \Phi^{5}= & (\{\text { ret }\},\{\chi \dot{\overline{\bar{F}}} \text { ret }\} \\
& \{\chi \dot{\overline{\bar{F}}} \text { ret }\})\end{aligned}$ & {$\left[\right.$ call, $\left.\Phi^{1}\right]\left[\right.$ call, $\left.\Phi^{0}\right] \perp$} & $\doteq$ \\
\hline 7 & ret $(\text { call })^{\omega}$ & $\Phi^{4}$ & {$\left[\right.$ ret,$\left.\Phi^{1}\right]\left[\right.$ call, $\left.\Phi^{0}\right] \perp$} & $\doteq$ \\
\hline 8 & $\operatorname{ret}(\text { call })^{\omega}$ & $\Phi^{6}=(\{$ ret $\},\{\chi \dot{\overline{\bar{F}}}$ ret $\}, \emptyset)$ & {$\left[\right.$ call, $\left.\Phi^{0}\right] \perp$} & $\doteq$ \\
\hline 9 & $(\text { call })^{\omega}$ & $\Phi^{7}=(\{$ call $\}, \emptyset, \emptyset)$ & {$\left[\right.$ ret,,$\left.\Phi^{0}\right] \perp$} & $>$ \\
\hline
\end{tabular}

Fig. 6. Prefix of an accepting run of the automaton for $\chi_{F}^{d}$ ret.

the satisfaction of its temporal requirement. Then, it is possible to define an acceptance set $F_{\chi \dot{\bar{F}} \psi} \in \mathbf{F}$, as the set of states not containing $\chi_{\bar{F}}^{\dot{\bar{F}}} \psi$ in any part. Figure 6 shows an $\omega$ OPBA run of this kind. Notice that after step $7 \chi \dot{\bar{F}} \psi$ does not appear in any state's in-stack part, so the run is accepting.

This construction is formalized as follows. Let $\psi \in C l_{\text {stack }}(\varphi)$. We add a few constraints on the transition relations. For any $\Phi, \Theta, \Psi \in Q_{\omega}$ and $a \in \mathcal{P}(A P)$ :

6. let $(\Phi, a, \Theta) \in \delta_{\text {push }}$ : if $\psi \in \Phi_{p}$, then $\psi \in \Theta_{s}$;

7. let $(\Phi, a, \Theta) \in \delta_{\text {push } / \text { shift }}$ : if $\psi \in \Phi_{s}$, then $\psi \in \Theta_{s}$;

8. let $(\Phi, \Theta, \Psi) \in \delta_{p o p}$ : if $\psi \in \Phi_{s}$ and $\psi \in \Theta_{s}$, then $\psi \in \Psi_{s}$.

An acceptance condition for summary until operators is also needed. For $\psi \mathcal{U}_{\chi}^{d} \theta \in C l(\varphi)$, we add an acceptance set $\mathbf{F}_{\psi \mathcal{U}_{\chi}^{d} \theta}$ such that for any $\Phi$ in it we

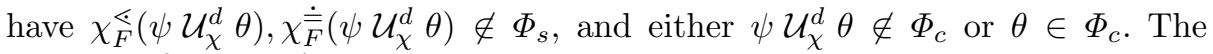
condition for $\psi \mathcal{U}_{\chi}^{u} \theta$ is symmetric.

\subsection{Complexity}

The set $C l(\varphi)$ is linear in $|\varphi|$, the length of $\varphi$. Atoms $(\varphi)$ has size at most $2^{|C l(\varphi)|}=2^{O(|\varphi|)}$, and the size of the set of states is the square of that in the finite case, and is bounded by its cube in the $\omega$-case. Moreover, the use of the equivalences for the hierarchical operators causes only a linear increase in the length of $\varphi$. Therefore,

Theorem 2. Given a POTL formula $\varphi$, it is possible to build an OPA or an $\omega O P B A \mathcal{A}_{\varphi}$ accepting the language denoted by $\varphi$ with at most $2^{O(|\varphi|)}$ states.

$\mathcal{A}_{\varphi}$ can then be intersected [42] with an OPA/ $\omega$ OPBA modeling a program (e.g. Fig. 2), and emptiness can be decided with summarization techniques [4]. 
Table 1. Results of the evaluation. '\# states' refers to the OPA to be verified.

\begin{tabular}{l|l|l|r|r|r|l}
\hline \multirow{2}{*}{ Benchmark name } & \multirow{2}{*}{ \# states } & Time $(\mathrm{ms})$ & \multicolumn{3}{|l}{ Memory $(\mathrm{KiB})$} & \multirow{2}{*}{ Result } \\
\cline { 4 - 6 } & & & Total & MC only & \\
\hline 1 & Generic (Fig. 2) & 12 & 867 & 70,040 & 10,166 & True \\
\hline 2 & Generic medium & 24 & 673 & 70,064 & 4,043 & False \\
\hline 3 & Generic larger & 30 & 1,014 & 70,063 & 14,160 & True \\
\hline 4 & Jensen & 42 & 305 & 70,050 & 3,154 & True \\
\hline 5 & Unsafe stack & 63 & 1,493 & 109,610 & 43,177 & False \\
\hline 6 & Safe stack & 77 & 637 & 70,089 & 7,234 & True \\
\hline 7 & Unsafe stack neutrality & 63 & 5,286 & 383,312 & 167,654 & True \\
\hline 8 & Safe stack neutrality & 77 & 840 & 70,077 & 16,773 & True \\
\hline
\end{tabular}

\section{Experimental Evaluation}

We implemented the OPA construction of Sect. 4 in an explicit-state model checking tool called POMC. The tool is written in Haskell [45], a purely functional, statically typed programming language with lazy evaluation. POMC checks OPA for emptiness by checking the reachability of an accepting configuration, by means of a modified DFS of the transition relation. This algorithm, similar to the one in [9], exploits the fact that all transitions only consider the topmost stack symbol, so reachability is actually computed only for semi-configurations made of one stack symbol and one state. Each time a chain support is explored, its ending semi-configuration is saved and associated with the starting one, so the next time the latter is reached, the support does not have to be re-explored. This allows the algorithm to exploit the cyclicities of OPA to terminate after having explored the whole transition relation. Given a POTL specification $\varphi$ and an OPA $\mathcal{A}$ to be checked, POMC executes the reachability algorithm, generating the product between $\mathcal{A}$ and the OPA for $\neg \varphi$ on-the-fly. The present prototype of POMC only supports finite-word model checking; its extension to deal with $\omega$-languages is under development.

We checked with POMC several requirements on three case studies and we report the results in Table 1 . Some additional formulas we checked are in Table 2. Such results can be reproduced through a publicly available artifact. ${ }^{2}$ The experiments were executed on a laptop with a $2.2 \mathrm{GHz}$ Intel processor and $15 \mathrm{GiB}$ of RAM, running Ubuntu GNU/Linux 20.04. In the tables, by "Total" memory we mean the maximum resident memory including the Haskell runtime (which allocates $70 \mathrm{MiB}$ by default), and by "MC only" the maximum memory used by model checking as reported by the runtime. Since model checking is polynomial in OPA size and exponential in formula length, we focus on checking a variety of requirements, rather than large OPA.

$\overline{2}$ https://doi.org/10.5281/zenodo.4723741. 
Generic Procedural Program. We checked formula

$$
\square\left(\left(\text { call } \wedge \mathrm{p}_{B} \wedge \operatorname{Scall}\left(\top, \mathrm{p}_{A}\right)\right) \Longrightarrow \operatorname{CallThr}(\top)\right)
$$

from Sect. 3.1 on the OPA of Fig. 2 (bench. 1), and also against two larger OPA (2, where the property does not hold, and 3 , where it holds).

We also checked the largest of such OPA against a set of formulas devised with the purpose of testing all POTL operators. The results are reported in Table 2. All formulas are checked very quickly, with only one outlier that runs out of memory. We ran the same experiment on a machine with a $2.0 \mathrm{GHz} \mathrm{AMD}$ CPU and $512 \mathrm{GiB}$ of RAM running Debian GNU/Linux 10, obtaining a time of $367 \mathrm{~s}$ with a memory occupancy of $16.3 \mathrm{GiB}$.

Stack Inspection. The security framework of the Java Development Kit (JDK) is based on stack inspection, i.e. the analysis of the contents of the program's stack during the execution. The JDK provides method checkPermission (perm) from class AccessController, which searches the stack for frames of functions that have not been granted permission perm. If any are found, an exception is thrown. Such permission checks prevent the execution of privileged code by unauthorized parts of the program, but they must be placed in sensitive points manually. Failure to place them appropriately may cause the unauthorized execution of privileged code. An automated tool to check that no code can escape such checks is thus desirable. Any such tool would need the ability to model exceptions, as they are used to avoid code execution in case of security violations.

[37] explains such needs by providing an example Java program for managing a bank account. It allows the user to check the account balance, and to withdraw money. To perform such tasks, the invoking program must have been granted permissions CanPay and Debit, respectively. We modeled such program as an OPA (4), and proved that the program enforces such security measures effectively by checking it against the formula

$$
\square\left(\text { call } \wedge \text { read } \Longrightarrow \neg\left(\top \mathcal{S}_{\chi}^{d}(\text { call } \wedge \neg \text { CanPay } \wedge \neg \text { read })\right)\right)
$$

meaning that the account balance cannot be read if some function in the stack lacks the CanPay permission (a similar formula checks the Debit permission).

Exception Safety. [53] is a tutorial on how to make exception-safe generic containers in $\mathrm{C}++$. It presents two implementations of a generic stack data structure, parametric on the element type $\mathrm{T}$. The first one is not exception-safe: if the constructor of $\mathrm{T}$ throws an exception during a pop action, the topmost element is removed, but it is not returned, and it is lost. This violates the strong exception safety requirement that each operation is rolled back if an exception is thrown. The second version of the data structure instead satisfies such requirement.

While exception safety is, in general, undecidable, it is possible to prove the stronger requirement that each modification to the data structure is only committed once no more exceptions can be thrown. We modeled both versions as OPA, and checked such requirement with the following formula:

$$
\square\left(\text { exc } \Longrightarrow \neg\left(\left(\ominus^{u} \text { modified } \vee \chi_{P}^{u} \text { modified }\right) \wedge \chi_{P}^{u}(\text { Stack }:: \text { push } \vee \text { Stack :: pop })\right)\right)
$$


POMC successfully found a counterexample for the first implementation (5), and proved the safety of the second one (6).

Additionally, we proved that both implementations are exception neutral (7, 8), i.e. Stack functions do not block exceptions thrown by the underlying type T. This was accomplished by checking the following formula:

$$
\square\left(\text { exc } \wedge \ominus^{u} \mathrm{~T} \wedge \chi_{P}^{d}\left(\text { han } \wedge \chi_{P}^{d} \text { Stack }\right) \Longrightarrow \chi_{P}^{d} \chi_{P}^{d} \chi_{F}^{u} \text { exc }\right)
$$

Table 2. Results of the additional experiments on OPA "generic larger".

\begin{tabular}{|c|c|c|c|c|}
\hline \multirow[t]{2}{*}{ Formula } & \multirow{2}{*}{\begin{tabular}{|l|} 
Time \\
$(\mathrm{ms})$
\end{tabular}} & \multicolumn{2}{|c|}{ Memory (KiB) } & \multirow[t]{2}{*}{ Result } \\
\hline & & Tot. & $\mathrm{MC}$ & \\
\hline$\chi_{F}^{d} \mathrm{p}_{E r r}$ & 1.1 & 70,095 & 175 & False \\
\hline$\bigcirc^{d}\left(\bigcirc^{d}\left(\right.\right.$ call $\wedge \chi_{F}^{u}$ exc $\left.)\right)$ & 21.0 & 70,095 & 1,290 & False \\
\hline$\bigcirc^{d}\left(\right.$ han $\wedge\left(\chi_{F}^{d}\left(\operatorname{exc} \wedge \chi_{P}^{u}\right.\right.$ call $\left.\left.)\right)\right)$ & 42.2 & 70,088 & 2,297 & False \\
\hline$\square\left(\right.$ exc $\Longrightarrow \chi_{P}^{u}$ call $)$ & 10.7 & 70,099 & 839 & True \\
\hline$\top \mathcal{U}_{\chi}^{d}$ exc & 2.2 & 70,093 & 121 & False \\
\hline$\bigcirc^{d}\left(\bigcirc^{d}\left(\top \mathcal{U}_{\chi}^{d}\right.\right.$ exc $\left.)\right)$ & 4.3 & 70,094 & 113 & False \\
\hline$\square\left(\left(\right.\right.$ call $\wedge \mathrm{p}_{A} \wedge\left(\neg\right.$ ret $\left.\left.\mathcal{U}_{\chi}^{d} \mathrm{WRx}\right)\right) \Longrightarrow \chi_{F}^{u}$ exc $)$ & $3,257.7$ & 238,833 & 102,582 & True \\
\hline$\bigcirc^{d}\left(\bigcirc^{u}\right.$ call $)$ & 0.7 & 70,094 & 139 & False \\
\hline$\bigcirc^{d}\left(\bigcirc^{d}\left(\bigcirc^{d}\left(\bigodot^{u}\right.\right.\right.$ call $\left.\left.)\right)\right)$ & 3.4 & 70,108 & 126 & False \\
\hline$\chi_{F}^{d}\left(\bigcirc^{d}\left(\Theta^{u}\right.\right.$ call $\left.)\right)$ & 1.3 & 70,096 & 137 & False \\
\hline$\square\left(\left(\right.\right.$ call $\left.\left.\wedge \mathrm{p}_{A} \wedge \operatorname{CallTh} r(\top)\right) \Longrightarrow \operatorname{CallTh} r\left(\mathrm{e}_{B}\right)\right)$ & $7,793.7$ & 402,420 & 173,639 & False \\
\hline$\diamond\left(\bigcirc_{H}^{d} \mathrm{p}_{B}\right)$ & 2.1 & 70,097 & 114 & False \\
\hline$\diamond\left(\ominus_{H}^{d} \mathrm{p}_{B}\right)$ & 2.8 & 70,097 & 114 & False \\
\hline$\diamond\left(\mathrm{p}_{A} \wedge\left(\right.\right.$ call $\left.\left.\mathcal{U}_{H}^{d} \mathrm{p}_{C}\right)\right)$ & 594.9 & 77,806 & 29,786 & True \\
\hline$\diamond\left(\mathrm{p}_{C} \wedge\left(\right.\right.$ call $\left.\left.\mathcal{S}_{H}^{d} \mathrm{p}_{A}\right)\right)$ & 676.6 & 96,296 & 37,949 & True \\
\hline$\square\left(\left(\mathrm{p}_{C} \wedge \chi_{F}^{u}\right.\right.$ exc $\left.) \Longrightarrow\left(\neg \mathrm{p}_{A} \mathcal{S}_{H}^{d} \mathrm{p}_{B}\right)\right)$ & - & - & - & OOM \\
\hline$\square\left(\right.$ call $\left.\wedge \mathrm{p}_{B} \Longrightarrow \neg \mathrm{p}_{C} \mathcal{U}_{H}^{u} \mathrm{p}_{E r r}\right)$ & 198.2 & 70,088 & 10,606 & True \\
\hline$\diamond\left(\bigcirc_{H}^{u} \mathrm{p}_{E r r}\right)$ & 1.1 & 70,093 & 114 & False \\
\hline$\diamond\left(\ominus_{H}^{u} \mathrm{p}_{E r r}\right)$ & 1.2 & 70,089 & 114 & False \\
\hline$\diamond\left(\mathrm{p}_{A} \wedge\left(\right.\right.$ call $\left.\left.\mathcal{U}_{H}^{u} \mathrm{p}_{B}\right)\right)$ & 10.3 & 70,105 & 115 & False \\
\hline$\diamond\left(\mathrm{p}_{B} \wedge\left(\right.\right.$ call $\left.\left.\mathcal{S}_{H}^{u} \mathrm{p}_{A}\right)\right)$ & 10.8 & 70,095 & 115 & False \\
\hline$\square\left(\right.$ call $\Longrightarrow \chi_{F}^{d}$ ret $)$ & 3.0 & 70,095 & 112 & False \\
\hline$\square\left(\right.$ call $\Longrightarrow \neg \bigcirc^{u}$ exc $)$ & 1.9 & 70,106 & 113 & False \\
\hline$\square\left(\right.$ call $\wedge \mathrm{p}_{A} \Longrightarrow \neg$ CallThr $\left.(\top)\right)$ & 110.7 & 70,094 & 4,937 & False \\
\hline$\square\left(\right.$ exc $\Longrightarrow \neg\left(\Theta^{u}\left(\right.\right.$ call $\left.\wedge p_{A}\right) \vee \chi_{P}^{u}\left(\right.$ call $\left.\left.\left.\wedge p_{A}\right)\right)\right)$ & 28.9 & 70,095 & 112 & False \\
\hline$\square\left(\left(\right.\right.$ call $\wedge \mathrm{p}_{B} \wedge\left(\right.$ call $\mathcal{S}_{\chi}^{d}\left(\right.$ call $\left.\left.\left.\wedge \mathrm{p}_{A}\right)\right)\right) \Longrightarrow \operatorname{CallTh} r(\mathrm{~T})$ & 926.1 & 70,104 & 13,310 & True \\
\hline$\square\left(\right.$ han $\Longrightarrow \chi_{F}^{u}$ ret $)$ & 17.0 & 70,079 & 1,252 & True \\
\hline$\top \mathcal{U}_{\chi}^{u}$ exc & 7.7 & 70,101 & 121 & True \\
\hline$\bigcirc^{d}\left(\bigcirc^{d}\left(\top \mathcal{U}_{\chi}^{u}\right.\right.$ exc $\left.)\right)$ & 44.6 & 70,104 & 2,376 & True \\
\hline$\bigcirc^{d}\left(\bigcirc^{d}\left(\bigcirc^{d}\left(\top \mathcal{U}_{\chi}^{u}\right.\right.\right.$ exc $\left.\left.)\right)\right)$ & 123.7 & 70,090 & 5,261 & False \\
\hline$\square\left(\right.$ call $\wedge \mathrm{p}_{C} \Longrightarrow\left(T \mathcal{U}_{\chi}^{u}\right.$ exc $\wedge \chi_{P}^{d}$ han $\left.)\right)$ & 92.9 & 70,096 & 1,346 & False \\
\hline call $\mathcal{U}_{\chi}^{d}\left(\right.$ ret $\left.\wedge \mathrm{p}_{E r r}\right)$ & 1.8 & 70,107 & 114 & False \\
\hline$\chi_{F}^{d}\left(\right.$ call $\wedge\left((\right.$ call $\vee$ exc $\left.\left.) \mathcal{S}_{\chi}^{u} \mathrm{p}_{B}\right)\right)$ & 10.8 & 70,086 & 117 & False \\
\hline$\bigcirc^{d}\left(\bigcirc^{d}\left((\right.\right.$ call $\vee$ exc $) \mathcal{U}_{\chi}^{u}$ ret $\left.)\right)$ & 5.3 & 70,094 & 114 & False \\
\hline
\end{tabular}




\section{Conclusions}

We introduced the temporal logic POTL, gave an automata-theoretic model checking procedure, and implemented it in a prototype tool. The results obtained in its experimental evaluation are promising. Additionally, POTL is proved to be FO-complete in a technical report [23]. We argue that the strong gain in expressive power w.r.t. previous approaches to model checking CFL, which comes without an increase in computational complexity, is worth the technicalities needed to achieve the present - and future - results.

In the evaluation, we used models directly coded into OPAs. To ease user interaction with our tool, we additionally implemented a new input format based on a simple procedural language with exceptions and Boolean variables, which is automatically translated into OPA. Moreover, we are currently working on the implementation of the model checking for $\omega$-words, described in Sect. 4.1.

As a future research step, we plan to develop user-friendly domain-specific languages for specification too, to prove that OP languages and logics are suitable in practice to program verification.

Acknowledgments. We are thankful to Davide Bergamaschi for developing an early POMC prototype, and to Francesco Pontiggia for implementing performance optimizations.

\section{References}

1. Abrahams, D.: Exception-Fsaety in generic components. In: Jazayeri, M., Loos, R.G.K., Musser, D.R. (eds.) Generic Programming. LNCS, vol. 1766, pp. 69-79. Springer, Heidelberg (2000). https://doi.org/10.1007/3-540-39953-4_6

2. Alur, R., Arenas, M., Barceló, P., Etessami, K., Immerman, N., Libkin, L.: Firstorder and temporal logics for nested words. LMCS 4(4), 1-44 (2008)

3. Alur, R., Benedikt, M., Etessami, K., Godefroid, P., Reps, T., Yannakakis, M.: Analysis of recursive state machines. ACM Trans. Program. Lang. Syst. 27(4), 786-818 (2005). https://doi.org/10.1145/1075382.1075387

4. Alur, R., Bouajjani, A., Esparza, J.: Model checking procedural programs. Handbook of Model Checking, pp. 541-572. Springer, Cham (2018). https://doi.org/10. 1007/978-3-319-10575-8_17

5. Alur, R., Chaudhuri, S., Madhusudan, P.: Software model checking using languages of nested trees. ACM Trans. Program. Lang. Syst. 33(5), 15:1-15:45 (2011)

6. Alur, R., Etessami, K., Madhusudan, P.: A temporal logic of nested calls and returns. In: Jensen, K., Podelski, A. (eds.) TACAS 2004. LNCS, vol. 2988, pp. 467-481. Springer, Heidelberg (2004). https://doi.org/10.1007/978-3-540-24730$2 \_35$

7. Alur, R., Madhusudan, P.: Visibly pushdown languages. In: ACM STOC (2004)

8. Alur, R., Madhusudan, P.: Adding nesting structure to words. JACM 56(3), 1-43 (2009)

9. Alur, R., Chaudhuri, S., Etessami, K., Madhusudan, P.: On-the-fly reachability and cycle detection for recursive state machines. In: Halbwachs, N., Zuck, L.D. (eds.) TACAS 2005. LNCS, vol. 3440, pp. 61-76. Springer, Heidelberg (2005). https:// doi.org/10.1007/978-3-540-31980-1_5 
10. Ball, T., Rajamani, S.K.: Bebop: a symbolic model checker for Boolean programs. In: Havelund, K., Penix, J., Visser, W. (eds.) SPIN 2000. LNCS, vol. 1885, pp. 113-130. Springer, Heidelberg (2000). https://doi.org/10.1007/10722468_7

11. Barenghi, A., Crespi Reghizzi, S., Mandrioli, D., Panella, F., Pradella, M.: Parallel parsing made practical. Sci. Comput. Program. 112, 195-226 (2015). https://doi. org/10.1016/j.scico.2015.09.002

12. Bouajjani, A., Echahed, R., Habermehl, P.: On the verification problem of nonregular properties for nonregular processes. LICS 95, 123-133 (1995)

13. Bouajjani, A., Esparza, J., Maler, O.: Reachability analysis of pushdown automata: application to model-checking. In: Mazurkiewicz, A., Winkowski, J. (eds.) CONCUR 1997. LNCS, vol. 1243, pp. 135-150. Springer, Heidelberg (1997). https:// doi.org/10.1007/3-540-63141-0_10

14. Bouajjani, A., Habermehl, P.: Constrained properties, semilinear systems, and Petri nets. In: Montanari, U., Sassone, V. (eds.) CONCUR 1996. LNCS, vol. 1119, pp. 481-497. Springer, Heidelberg (1996). https://doi.org/10.1007/3-540-61604$7 \_71$

15. Bozzelli, L., Murano, A., Peron, A.: Timed context-free temporal logics. In: GandALF 2018. EPTCS, vol. 277, pp. 235-249. Open Publishing Association (2018). https://doi.org/10.4204/EPTCS.277.17

16. Bozzelli, L., Sánchez, C.: Visibly linear temporal logic. In: Demri, S., Kapur, D., Weidenbach, C. (eds.) IJCAR 2014. LNCS (LNAI), vol. 8562, pp. 418-433. Springer, Cham (2014). https://doi.org/10.1007/978-3-319-08587-6_33

17. Burkart, O., Steffen, B.: Model checking the full modal mu-calculus for infinite sequential processes. Theor. Comput. Sci. 221(1-2), 251-270 (1999). https://doi. org/10.1016/S0304-3975(99)00034-1

18. Chatterjee, K., Ma, D., Majumdar, R., Zhao, T., Henzinger, T.A., Palsberg, J.: Stack size analysis for interrupt-driven programs. Inf. Comput. 194(2), 144-174 (2004). https://doi.org/10.1016/j.ic.2004.06.001

19. Chaudhuri, S., Alur, R.: Instrumenting $\mathrm{C}$ programs with nested word monitors. In: Bošnački, D., Edelkamp, S. (eds.) SPIN 2007. LNCS, vol. 4595, pp. 279-283. Springer, Heidelberg (2007). https://doi.org/10.1007/978-3-540-73370-6_20

20. Chen, F., Roşu, G.: Java-MOP: a monitoring oriented programming environment for Java. In: Halbwachs, N., Zuck, L.D. (eds.) TACAS 2005. LNCS, vol. 3440, pp. 546-550. Springer, Heidelberg (2005). https://doi.org/10.1007/978-3-540-31980$1 \_36$

21. Chiari, M., Mandrioli, D., Pradella, M.: POTL: a first-order complete temporal logic for operator precedence languages. CoRR abs/1910.09327 (2019). http:// arxiv.org/abs/1910.09327

22. Chiari, M., Mandrioli, D., Pradella, M.: Operator precedence temporal logic and model checking. Theor. Comput. Sci. 848, 47-81 (2020). https://doi.org/10.1016/ j.tcs.2020.08.034

23. Chiari, M., Mandrioli, D., Pradella, M.: A first-order complete temporal logic for structured context-free languages. CoRR abs/2105.10740 (2021). https://arxiv. org/abs/2105.10740

24. Clarke, E.M., Henzinger, T.A., Veith, H., Bloem, R. (eds.): Handbook of Model Checking. Springer, Heidelberg (2018). https://doi.org/10.1007/978-3-31910575-8

25. Crespi Reghizzi, S., Mandrioli, D.: Operator precedence and the visibly pushdown property. JCSS 78(6), 1837-1867 (2012). https://doi.org/10.1016/j.jcss.2011.12. 006 
26. D'Antoni, L.: A symbolic automata library. https://github.com/lorisdanto/ symbolicautomata

27. Driscoll, E., Thakur, A., Reps, T.: OpenNWA: a nested-word automaton library. In: Madhusudan, P., Seshia, S.A. (eds.) CAV 2012. LNCS, vol. 7358, pp. 665-671. Springer, Heidelberg (2012). https://doi.org/10.1007/978-3-642-31424-7_47

28. Esparza, J., Hansel, D., Rossmanith, P., Schwoon, S.: Efficient algorithms for model checking pushdown systems. In: Emerson, E.A., Sistla, A.P. (eds.) CAV 2000. LNCS, vol. 1855, pp. 232-247. Springer, Heidelberg (2000). https://doi.org/10. 1007/10722167_20

29. Esparza, J., Kučera, A., Schwoon, S.: Model checking LTL with regular valuations for pushdown systems. Inf. Comput. 186(2), 355-376 (2003)

30. Finkel, A., Willems, B., Wolper, P.: A direct symbolic approach to model checking pushdown systems. In: Infinity 1997. ENTCS, vol. 9, pp. 27-37. Elsevier (1997). https://doi.org/10.1016/S1571-0661(05)80426-8

31. Floyd, R.W.: Syntactic analysis and operator precedence. JACM 10(3), 316-333 (1963). https://doi.org/10.1145/321172.321179

32. Godefroid, P., Yannakakis, M.: Analysis of Boolean programs. In: Piterman, N., Smolka, S.A. (eds.) TACAS 2013. LNCS, vol. 7795, pp. 214-229. Springer, Heidelberg (2013). https://doi.org/10.1007/978-3-642-36742-7_16

33. Grune, D., Jacobs, C.J.: Parsing Techniques: A Practical Guide. Springer, New York (2008). https://doi.org/10.1007/978-0-387-68954-8

34. Harel, D., Kozen, D., Tiuryn, J.: Dynamic logic. In: Gabbay, D.M., Guenthner, F. (eds.) Handbook of Philosophical Logic. Handbook of Philosophical Logic, vol. 4. Springer, Dordrecht (2001). https://doi.org/10.1007/978-94-017-0456-4_2

35. Harrison, M.A.: Introduction to Formal Language Theory. Addison Wesley, Boston (1978)

36. Henzinger, T.A., Jhala, R., Majumdar, R., Sutre, G.: Software verification with BLAST. In: Ball, T., Rajamani, S.K. (eds.) SPIN 2003. Software verification with BLAST, vol. 2648, pp. 235-239. Springer, Heidelberg (2003). https://doi.org/10. 1007/3-540-44829-2_17

37. Jensen, T., Le Metayer, D., Thorn, T.: Verification of control flow based security properties. In: Proceedings of 1999 IEEE Symposium on Security and Privacy, pp. 89-103 (1999). https://doi.org/10.1109/SECPRI.1999.766902

38. Jhala, R., Podelski, A., Rybalchenko, A.: Predicate abstraction for program verification. Handbook of Model Checking, pp. 447-491. Springer, Cham (2018). https://doi.org/10.1007/978-3-319-10575-8_15

39. Kamp, H.: Tense logic and the theory of linear order. Ph.D. thesis, University of California, Los Angeles (1968)

40. Kupferman, O., Piterman, N., Vardi, M.Y.: Model checking linear properties of prefix-recognizable systems. In: Brinksma, E., Larsen, K.G. (eds.) CAV 2002. LNCS, vol. 2404, pp. 371-385. Springer, Heidelberg (2002). https://doi.org/10. 1007/3-540-45657-0_31

41. Kupferman, O., Piterman, N., Vardi, M.Y.: Pushdown specifications. In: Baaz, M., Voronkov, A. (eds.) LPAR 2002. LNCS (LNAI), vol. 2514, pp. 262-277. Springer, Heidelberg (2002). https://doi.org/10.1007/3-540-36078-6_18

42. Lonati, V., Mandrioli, D., Panella, F., Pradella, M.: Operator precedence languages: their automata-theoretic and logic characterization. SIAM J. Comput. 44(4), 1026-1088 (2015). https://doi.org/10.1137/140978818

43. Mandrioli, D., Pradella, M.: Generalizing input-driven languages: theoretical and practical benefits. Comput. Sci. Rev. 27, 61-87 (2018). https://doi.org/10.1016/j. cosrev.2017.12.001 
44. Mandrioli, D., Pradella, M., Crespi Reghizzi, S.: Star-freeness, first-order definability and aperiodicity of structured context-free languages. In: Pun, V.K.I., Stolz, V., Simao, A. (eds.) ICTAC 2020. LNCS, vol. 12545, pp. 161-180. Springer, Cham (2020). https://doi.org/10.1007/978-3-030-64276-1_9

45. Marlow, S.: Haskell 2010 language report (2010). https://www.haskell.org/ onlinereport/haskell2010/

46. McNaughton, R.: Parenthesis grammars. JACM 14(3), 490-500 (1967)

47. Mehlhorn, K.: Pebbling mountain ranges and its application to DCFL-recognition. In: de Bakker, J., van Leeuwen, J. (eds.) ICALP 1980. LNCS, vol. 85, pp. 422-435. Springer, Heidelberg (1980). https://doi.org/10.1007/3-540-10003-2_89

48. Nguyen, H.: Visibly pushdown automata library (2006). https://web.imtatlantique.fr/x-info/hnguyen/vpa

49. Nguyen, H., Touili, T.: CARET model checking for malware detection. In: SPIN 2017, pp. 152-161. ACM (2017). https://doi.org/10.1145/3092282.3092301

50. Nguyen, H., Touili, T.: CARET model checking for pushdown systems. In: SAC 2017, pp. 1393-1400. ACM (2017). https://doi.org/10.1145/3019612.3019829

51. Piterman, N., Vardi, M.Y.: Global model-checking of infinite-state systems. In: Alur, R., Peled, D.A. (eds.) CAV 2004. LNCS, vol. 3114, pp. 387-400. Springer, Heidelberg (2004). https://doi.org/10.1007/978-3-540-27813-9_30

52. Roşu, G., Chen, F., Ball, T.: Synthesizing monitors for safety properties: this time with calls and returns. In: Leucker, M. (ed.) RV 2008. LNCS, vol. 5289, pp. 51-68. Springer, Heidelberg (2008). https://doi.org/10.1007/978-3-540-89247-2_4

53. Sutter, H.: Exception-safe generic containers. C++ Report (1997). https:// ptgmedia.pearsoncmg.com/imprint_downloads/informit/aw/meyerscddemo/ DEMO/MAGAZINE/SU_FRAME.HTM

54. Tang, N.V., Ohsaki, H.: Checking on-the-fly universality and inclusion problems of visibly pushdown automata. IEICE Trans. Fundam. Electron. Commun. Comput. Sci. 94-A(12), 2794-2801 (2011). https://doi.org/10.1587/transfun.E94.A.2794

55. Walukiewicz, I.: Pushdown processes: games and model-checking. Inf. Comput. 164(2), 234-263 (2001). https://doi.org/10.1006/inco.2000.2894

Open Access This chapter is licensed under the terms of the Creative Commons Attribution 4.0 International License (http://creativecommons.org/licenses/by/4.0/), which permits use, sharing, adaptation, distribution and reproduction in any medium or format, as long as you give appropriate credit to the original author(s) and the source, provide a link to the Creative Commons license and indicate if changes were made.

The images or other third party material in this chapter are included in the chapter's Creative Commons license, unless indicated otherwise in a credit line to the material. If material is not included in the chapter's Creative Commons license and your intended use is not permitted by statutory regulation or exceeds the permitted use, you will need to obtain permission directly from the copyright holder.

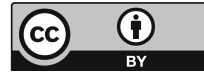

